\title{
A posteriori error estimates for the coupling equations of scalar conservation laws
}

\author{
Mohammad Izadi
}

Received: 19 February 2008 / Accepted: 14 October 2009 / Published online: 14 November 2009

(C) The Author(s) 2009. This article is published with open access at Springerlink.com

\begin{abstract}
In this paper we prove a posteriori $L_{2}\left(L_{2}\right)$ and $L_{\infty}\left(H^{-1}\right)$ residual based error estimates for a finite element method for the one-dimensional time dependent coupling equations of two scalar conservation laws. The underlying discretization scheme is Characteristic Galerkin method which is the particular variant of the Streamline diffusion finite element method for $\delta=0$. Our estimate contains certain strong stability factors related to the solution of an associated linearized dual problem combined with the Galerkin orthogonality of the finite element method. The stability factor measures the stability properties of the linearized dual problem. We compute the stability factors for some examples by solving the dual problem numerically.
\end{abstract}

Keywords A posteriori error estimates - Coupling equations · Dual problem · Finite element methods

Mathematics Subject Classification (2000) 65 N12 $\cdot 65$ N15 $\cdot 65 N 30 \cdot 76$ N10

\section{Introduction}

This paper is the second part in a series of two papers concerning approximate solutions for the coupling equations. In the first part [12], we derived, for smooth solutions in the Sobolev space $H^{k+1}$ of functions with their partial derivatives up to order

\footnotetext{
Communicated by Anders Szepessy.

M. Izadi $(\bowtie)$

Department of Mathematics, Institute for Advanced Studies in Basic Sciences (IASBS),

P.O. Box 45195-1159, Zanjan, Iran

e-mail: izadi@mis.mpg.de

Present address:

M. Izadi

Max Planck Institute for Mathematics in the Sciences, Inselstr. 22-26, 04103 Leipzig, Germany
} 
$k+1$ in $L_{2}$, optimal a priori error estimates for the Streamline diffusion finite element methods (for short, the Sd-method below) of order $\mathcal{O}\left(h^{k+1 / 2}\right)$. In this part we extend our studies to a posteriori estimates (see [1, 2, 6-9]) dealing with the following basic problem: To construct an algorithm for the numerical solution of the coupling equations such that the error between the exact and approximate solutions, measured in some appropriate norms such as weighted $L_{2}\left(L_{2}\right)$ and $L_{\infty}\left(H^{-1}\right)$ norms, is guaranteed to be below a given tolerance and such that the computational cost is almost minimal. These two properties are referred to as the reliability and efficiency of the algorithm, respectively. The a posteriori error analyzes are required for the reliability in the sense that the error is controlled by certain norms of the residual (which is obtained by inserting the computed finite element solution into the differential equation) term, whereas the a priori error estimates are based on controlling the size of the error by some norm of the unknown solution itself. As for the efficiency the adaptivity may be invoked to avoid unnecessary mesh-refinements on the regions where the contribution to the error is already small.

The main objective in this study is to derive a posteriori error estimates and prove strong stability estimates of the dual problem for the solution of the interface problem in a system of two different partial differential equations describing e.g. multifluids with different state equations and moving contact discontinuities. More specifically we consider the following coupling of two conservation laws in one dimension: Find $u:(x, t) \in \mathbb{R} \times \mathbb{R}_{+} \rightarrow u(x, t) \in \mathbb{R}$ such that

$$
\begin{cases}u_{t}+f_{R}(u)_{x}-(\varepsilon u)_{x x}=0, & x>0, t>0, \\ u_{t}+f_{L}(u)_{x}-(\varepsilon u)_{x x}=0, & x<0, t>0, \\ u(x, 0)=u_{0}, & x \in \mathbb{R},\end{cases}
$$

and also a suitable "continuity" condition

$$
u(x, t)=u^{b}(t), \quad t \geq 0,
$$

at the interface $x=0$, to be compatible with initial condition $u_{0}$ (for more details see $[10,12])$, where $\varepsilon=\varepsilon(x, t)$ is a positive small viscosity, $u_{0}: \mathbb{R} \rightarrow \mathbb{R}$ is a given function and $f_{\alpha}: \mathbb{R} \rightarrow \mathbb{R}, \alpha=L, R$, denote two "smooth" functions.

This is a system of nonlinear conservation laws arising in the study of fluid problems with two different equations on each side of the interface which may be fixed or are moving with the flow. For instance, on one side of the interface, we assume that the flow obeys the rules of the isentropic system of gas dynamics while on the other side flow is arbitrary. A more complex problem in the case of having to couple the Navier-Stokes system with the Euler system of gas dynamics [3], as well as modeling certain plasma physical problems cf [10].

This paper is organized as follows: In Sect. 2, we construct a space-time discretization and formulate the Streamline diffusion method for the approximation solution of the coupled problem. Section 3 is devoted to the proof of a posteriori error estimates for the corresponding Characteristic Galerkin for the perturbed coupled problem in two different norms. In Sect. 4, we state and prove the analytical strong stability estimates for dual solution of our problem and finally, in our concluding Sect. 5 we 
present some computational results, with emphasis on computation of the stability factors by solving associated dual problem.

\section{Finite element discretization}

We formulate a finite element method for the first equation involving $f_{R}$ in (1.1). We use the Galerkin method with piecewise linear basis functions which are continuous in space and discontinuous in time, i.e. the Sd-method. To do this we first introduce some basic notation.

Let $\left\{0=t_{0}<t_{1}<\cdots<t_{N}=T\right\}$ be a partition of the interval $I=(0, T)$ into the subintervals $I_{n}=\left(t_{n}, t_{n+1}\right)$, with time steps $k_{n}=t_{n+1}-t_{n}, n=0,1, \ldots, N-1$ and introduce the corresponding space-time "slabs" $\mathbf{S}_{n}=\mathbb{R}_{+} \times I_{n}$. On each slab for $h>0$, we define a space-time mesh $T_{h}^{n}=\{K\}$ with space-time element $K$, as an quasi-uniform subdivision (see e.g., Ciarlet [5]) of the slab $\mathbf{S}_{n}$, i.e., for each $K \in T_{h}^{n}$ there is an inscribed circle in $K$ such that the ratio of the diameter of this circle and the diameter of $K$ is bounded below, independently of $K$ and $h$.

Let now $q$ be a positive integer and introduce the finite element space

$$
U_{h}^{n}=\left\{u \in H^{1}\left(\mathbf{S}_{n}\right):\left.u\right|_{K} \in P_{q}(K), K \in T_{h}^{n}\right\},
$$

where $P_{q}(K)$ denotes the set of polynomials on $K$ of degree less than or equal to $q$. Then we define the trial and test function spaces as the subspaces of $U_{h}^{n}$ by

$$
\mathcal{V}_{h}^{n}=\left\{v \in U_{h}^{n}:\left.v\right|_{\Gamma}=u_{h}^{b}\right\}, \quad \text { and } \quad \mathcal{W}_{h}^{n}=\left\{w \in U_{h}^{n}:\left.w\right|_{\Gamma}=0\right\},
$$

respectively. Here $u_{h}^{b}$ is the trace of a function in $U_{h}^{n}$ approximating $u^{b}$ on $\Gamma$ which is the boundary of $\Omega=\mathbb{R}_{+} \times(0, T)$. Note that we may assume that the functions $v(x, t) \in \mathcal{V}_{h}^{n}$ and $w(x, t) \in \mathcal{W}_{h}^{n}$ are vanishing for sufficiently large $|x|$.

Summing over $n$, taking all the slabs together we get the function spaces

$$
\mathcal{V}_{h}=\prod_{n=0}^{N-1} \mathcal{V}_{h}^{n}, \quad \mathcal{W}_{h}=\prod_{n=0}^{N-1} \mathcal{W}_{h}^{n}
$$

i.e., we seek an approximate solution $u_{h} \in \mathcal{V}_{h}$ such that for $n=0,1, \ldots, N-1$ we have that $u_{h} \mid \mathbf{S}_{n}=u_{h}^{n}$. We emphasize that the functions in $\mathcal{V}_{h}$ are continuous in $x$ and possibly discontinuous in $t$ at discrete time levels $t_{n}$. Similar properties are valid for $w_{h} \in \mathcal{W}_{h}$.

In order to write the Sd-method in a compact form suitable for analysis, and because the functions in $\mathcal{V}_{h}$ may be discontinuous in time, we introduce the jump terms $[v]$ across each time level by defining, for $x>0$ and $n=0,1, \ldots, N-1$,

$$
[v]\left(x, t_{n}\right)= \begin{cases}v_{+} & \text {if } n=0, \\ v_{+}-v_{-} & \text {if } n \neq 0,\end{cases}
$$

where

$$
v_{+}=\lim _{s \rightarrow 0+} v(x, t+s), \quad v_{-}=\lim _{s \rightarrow 0-} v(x, t+s),
$$


and also we use the following notation

$$
\langle u, v\rangle_{n}=\int_{\mathbb{R}_{+}} u\left(x, t_{n}\right) v\left(x, t_{n}\right) d x, \quad|v|_{n}=\langle v, v\rangle_{n}^{1 / 2} .
$$

\subsection{The Sd-method}

We start with the first equation of system (1.1) and reformulate it as the following initial boundary value problem: Find $u$ such that

$$
\begin{aligned}
\mathcal{L}_{\varepsilon} u \equiv u_{t}+f_{R}^{\prime}(u) u_{x}-(\varepsilon u)_{x x} & =0 \quad \text { in } \Omega, \\
u(0, t) & =u^{b}, \quad t \in(0, T), \\
u(x, 0) & =u_{0}, \quad x \in \mathbb{R}_{+},
\end{aligned}
$$

where $\varepsilon$ is a positive viscosity coefficient, which we typically assume to be a 'small' constant specified below. Our main goal is to derive a posteriori error estimates for the above problem based on the Sd-method. In the formulation of the Sd-method below, if the mesh size is not small enough, the given viscosity $\varepsilon$ will be replaced by an artificial viscosity $\hat{\varepsilon}$ depending on the computed solution $u_{h}$ and the mesh size $h$. Therefore in the a posteriori error analysis of (2.1) it is convenient to split the total error $e=u-u_{h}$ into two parts $e=\rho+\theta$ where $\rho=u-\hat{u}$ with $\hat{u}$ the solution of a perturbed continuous problem obtained by replacing $\varepsilon$ by $\hat{\varepsilon}$ in (2.1), and $\hat{e}:=\theta=\hat{u}-u_{h}$ is the discretization error related to applying the Sd-method (now without modifying the viscosity coefficient) to the perturbed problem. In this case, the a posteriori error estimates (also underlying adaptive algorithm) is, in the case of space discretization only, basically as follows:

$$
\|\hat{e}\|_{A} \leq S C_{i}\left\|\frac{h^{2}}{\hat{\varepsilon}} \mathcal{R}\left(u_{h}\right)\right\|_{B},
$$

where $\|.\|_{A}$ and $\|\cdot\|_{B}$ are some norms, e.g., an $L_{p}\left(L_{q}\right)$ in time-space or $L_{\infty}\left(H^{-1}\right)$ norm, $h$ is the mesh size, and $\mathcal{R}\left(u_{h}\right)=\mathcal{L}_{\hat{\varepsilon}} u_{h}$ is the residual obtained inserting the computed finite element solution into the perturbed differential equation. Further $C_{i}$ is an interpolation constant which depend on the shape of the elements, the local order of polynomial approximation and the choice of norms, but not on the particular solution being approximated or the mesh size, and $S$ is a stability factor which measures certain stability properties of an associated continuous linearized dual problem.

The Sd-method for (2.1) can now be formulated as follows: Find $u_{h}^{n} \in \mathcal{V}_{h}^{n}$, such that for $n=0,1, \ldots, N-1$

$$
\begin{gathered}
\left(u_{h, t}^{n}+f_{R}^{\prime}\left(u_{h}^{n}\right) u_{h, x}^{n}, v_{h}^{n}+\delta\left(v_{h, t}^{n}+f_{R}^{\prime}\left(u_{h}^{n}\right) v_{h, x}^{n}\right)\right)_{n}+\left(\hat{\varepsilon} u_{h, x}^{n}, v_{h, x}^{n}\right)_{n} \\
+\left\langle\left[u_{h}\right], v_{h,+}^{n}\right\rangle_{n}+\int_{\Gamma_{n}} u_{h,+}^{n} v_{h,+}^{n} d t=\int_{\Gamma_{n}} u^{b} v_{h,+}^{n} d t \quad \forall v_{h}^{n} \in \mathcal{W}_{h}^{n}
\end{gathered}
$$


where $u_{h,-}^{0}=u_{0},\left[u_{h}\right]=u_{h,+}^{n}-u_{h,-}^{n}$,

$$
\begin{aligned}
\hat{\varepsilon} & =\max \left(\varepsilon, C_{1} h\left|\mathcal{R}\left(u_{h}\right)\right| /\left|\nabla u_{h}\right|, C_{2} h^{3 / 2}\right), \\
\mathcal{R}\left(u_{h}\right) & =\left|u_{h, t}+f\left(u_{h}\right)_{x}\right|+\frac{\left|\left[u_{h}\right]\right|}{k_{n}} \quad \text { on } \mathbf{S}_{n}, \\
\delta & =\bar{C} h,
\end{aligned}
$$

with $h$ denoting the mesh size, $\bar{C}$ and $C_{i}$ 's are positive constants and $\Gamma_{n}:=\{0\} \times I_{n}$. The artificial viscosity $\hat{\varepsilon}$ acts in an implicit way to provide additional stability near to shocks $\left(\hat{\varepsilon} \sim C_{1} h\right)$, and less in smooth regions $\left(\hat{\varepsilon} \sim C_{2} h^{3 / 2}\right)$.

In order to estimate the error $e=u-u_{h}$, we also need to estimate $\rho=u-\hat{u}$. To control the $u-\hat{u}$, we may adaptively refine the mesh until $\hat{\varepsilon}=\varepsilon$, giving $u=\hat{u}$, or alternatively approximate $\hat{e}$ in terms of $\varepsilon-\hat{\varepsilon}$. In the a posteriori error estimates for the Sd-method (2.3), below for simplicity we assume that $u^{b} \equiv 0, \hat{\varepsilon}=\varepsilon$ is constant and that the function $h(x, t)=h$ is constant, for all $x, t$. Further we consider the following simplified version of the Sd-method with $\hat{\varepsilon}=\bar{C} h$ and $\delta=0$ (an error analysis for the Sd-method in the case $\delta \neq 0$ is given in $[14,17])$ : Find $u_{h} \in \mathcal{V}_{h}^{n}$, such that for $n=0,1, \ldots, N-1$

$$
\left(u_{h, t}+f_{R}\left(u_{h}\right)_{x}, v\right)_{n}+\left(\hat{\varepsilon} u_{h, x}, v_{x}\right)_{n}+\left\langle\left[u_{h}\right], v_{+}\right\rangle_{n}=0, \quad \forall v \in \mathcal{W}_{h}^{n}
$$

\section{A posteriori error analysis}

\subsection{Introduction}

The a posteriori approach tries to estimate the error of approximating a particular solution by using the information from computation. The foundation is a rigorous a posteriori error estimate which bound the error by computable quantities that depend on the "known computed" numerical solution rather than the unknown exact solution.

The standard a posteriori error estimates for time-dependent problem as presented in $[11,16]$ and [6] typically rely on Galerkin orthogonality, interpolation estimates and strong stability estimates for a suitable dual problem running backward in time with a desired error functional as initial data on the right hand side as key ingredient. In our approach to a posteriori error analysis, in addition to these ingrediences, we rely also on the concept of stability factor. Below we, especially, consider these issues in more details.

\subsection{The dual problem}

In order to obtain a representation of the error (see Sect. 3.4), we consider the following auxiliary problem, referred to as the linearized dual problem: Find $\varphi$ such that

$$
\mathcal{L}_{\hat{\varepsilon}}^{*} \varphi \equiv-\varphi_{t}-A^{T} \varphi_{x}-\hat{\varepsilon} \varphi_{x x}=\psi_{1} \quad \text { in } \Omega
$$




$$
\begin{gathered}
\varphi(0, t)=0, \quad t \in(0, T), \\
\varphi(x, T)=\psi_{2}, \quad x \in \mathbb{R}_{+},
\end{gathered}
$$

where

$$
A=\int_{0}^{1} f_{R}^{\prime}\left(s u+(1-s) u_{h}\right) d s
$$

and $\mathcal{L}_{\hat{\varepsilon}}^{*}$ denotes the adjoint of the operator $\mathcal{L}$ is defined in (2.1). Note that this problem is computed "backward", i.e. from $t_{N}$, where the initial data is given, to 0 . Depending on the choice of $\psi_{1}$ or $\psi_{2}$, we get estimates of different norms or functionals of the error.

\subsection{Notations}

We use the following notation

$$
\begin{aligned}
(u, v)_{n} & =\int_{\mathbf{S}_{n}} u v d x d t, \quad(u, v)_{Q}=\sum_{n=0}^{N-1}(u, v)_{n}, \\
\|\cdot\| & =\|\cdot\|_{L_{2}(Q)}=(u, v)_{Q}^{\frac{1}{2}}, \quad\|\cdot\|_{L_{p}}=\|u(., t)\|_{L_{p}(\mathbb{R})}, \\
\|\cdot\|_{L_{\infty}\left(L_{p}\right)} & =\sup _{t \in[0, T]}\|u(., t)\|_{L_{p}}, \quad\|u\|_{L_{1}\left(L_{2}\right)}=\int_{0}^{T}\|u(., t)\|_{L_{2}} d t, \\
\|u\|_{H^{-1}} & =\left\|v_{x}\right\|_{L_{2}}, \quad \text { where }-v_{x x}=u, v\left(x_{L}\right)=v\left(x_{R}\right)=0,\|g\|_{L_{2}^{\psi}}=\left\|\psi^{\frac{1}{2}} g\right\|_{L_{2}},
\end{aligned}
$$

where the computational domain is restricted to the interval $\left(x_{L}, x_{R}\right)$ and $\psi$ is positive weight function.

By $D_{1} u_{h}$ and $D_{2} u_{h}$ we denote "discrete second derivative" of $u$ defined by

$$
D_{1} u_{h}(x, t)=\max _{j=i, i-1}\left|\frac{\left[u_{h, x}\left(x_{j}^{n}, t\right)\right]}{h_{j}^{n}}\right|, \quad x \in\left(x_{i-1}^{n}, x_{i}^{n}\right), t \in I_{n},
$$

where $\left[v\left(x_{j}^{n}\right)\right]=\lim _{\zeta \rightarrow 0^{+}}\left(v\left(x_{j}^{n}+\zeta\right)-v\left(x_{j}^{n}-\zeta\right)\right)$

$$
\begin{array}{ll}
\left(D_{2} w, v\right)_{n}=-\left(w_{x}, v_{x}\right)_{n} & \forall v \in \mathcal{W}_{h}^{n}, \\
\left(D_{2}^{\hat{\varepsilon}} w, v\right)_{n}=-\left(\hat{\varepsilon} w_{x}, v_{x}\right)_{n} & \forall v \in \mathcal{W}_{h}^{n},
\end{array}
$$

where $D_{2}^{\hat{\varepsilon}}, D_{2}: \mathcal{W}_{h}^{n} \rightarrow \mathcal{W}_{h}^{n}$.

The a posteriori error estimates contain residual of the computed solution defined by

$$
\begin{aligned}
& \mathcal{R}_{0}=u_{h, t}+f_{R}\left(u_{h}\right)_{x}, \quad \mathcal{R}_{1}=D_{1} u_{h}, \quad \mathcal{R}_{2}=-D_{2}^{\hat{\varepsilon}} u_{h}, \\
& \mathcal{R}_{3}=\frac{\left(\mathcal{P}_{n}-\mathbb{I}\right) u_{h,-}^{n}}{k_{n}} \text { on } \mathbf{S}_{n}, \quad \mathcal{R}_{4}=\left(u_{h,+}^{n}-u_{h,-}^{n}\right) / k_{n} \quad \text { on } \mathbf{S}_{n},
\end{aligned}
$$


where $\mathbb{I}$ is identity operator and $\mathcal{P}_{n}: \mathbf{S}_{n} \rightarrow \mathcal{W}_{h}^{n}$ denote the $L_{2}$-projection defined by

$$
\left(\mathcal{P}_{n} u, v\right)_{n}=(u, v)_{n}, \quad \forall v \in \mathcal{W}_{h}^{n}
$$

We defined $\mathcal{P}$ and $\pi$ by

$$
\left.(\mathcal{P} \varphi)\right|_{\mathbf{S}_{n}}=\mathcal{P}_{n}\left(\varphi \mid \mathbf{S}_{n}\right), \quad \text { and }\left.\quad(\pi \varphi)\right|_{\mathbf{S}_{n}}=\pi_{n}\left(\left.\varphi\right|_{\mathbf{S}_{n}}\right)
$$

respectively, where $\pi_{n}: L_{2}\left(\mathbf{S}_{n}\right) \rightarrow \Pi_{0, n}=\left\{v \in L_{2}\left(\mathbf{S}_{n}\right): v(x, \cdot)\right.$ is constant on $I_{n}$, $\left.x \in \mathbb{R}_{+}\right\}$is defined by

$$
\left.\pi_{n} v\right|_{\mathbf{S}_{n}}=\frac{1}{k_{n}} \int_{I_{n}} v(\cdot, t) d t \quad \forall v \in \Pi_{0, n} .
$$

We note that $\mathcal{P} \pi=\pi \mathcal{P}$ since $\mathcal{P}_{n} \pi_{n}=\pi_{n} \mathcal{P}_{n}$ for $n=0,1, \ldots, N-1$.

\subsection{An a posteriori error estimates in $L_{2}\left(L_{2}\right)$}

Let us now prove a basic a posteriori error estimate in a weighted $L_{2}$-norm. We start by representing the error $\|\hat{e}\|_{L_{2}^{\psi^{-1}}(\Omega)}^{2}$ in terms of the dual solution $\varphi$ of (3.1) choosing $\psi_{1}=\psi^{-1} \hat{e}$ and $\psi_{2}=0$. Multiplying (3.1a) by $\hat{e}$ and integrating over each $\mathbf{S}_{n}$, integrating by parts, and summing over $n$, we obtain the following error representation formula:

$$
\begin{aligned}
\|\hat{e}\|_{L_{2}^{\psi^{-1}}(\Omega)}^{2} & =\left(\hat{e}, \psi^{-1} \hat{e}\right)_{\Omega}=\left(\hat{e}, \mathcal{L}_{\hat{\varepsilon}}^{*} \varphi\right)_{\Omega} \\
& =\sum_{n=0}^{N-1}\left\{\left(\hat{e}_{t}+(A \hat{e})_{x}, \varphi\right)_{n}+\left(\hat{\varepsilon} \hat{e}_{x}, \varphi_{x}\right)_{n}\right\}-\sum_{n=0}^{N-1}\left\langle\left[u_{h}\right], \varphi_{+}\right\rangle_{n} \\
& =-\sum_{n=0}^{N-1}\left\{\left(u_{h, t}+f_{R}\left(u_{h}\right)_{x}, \varphi\right)_{n}+\left(\hat{\varepsilon} u_{h, x}, \varphi_{x}\right)_{n}\right\}-\sum_{n=0}^{N-1}\left\langle\left[u_{h}\right], \varphi_{+}\right\rangle_{n} .
\end{aligned}
$$

So that using the Galerkin orthogonality with $\Psi=\pi \mathcal{P} \varphi \in V_{h}$ as an suitable interpolant of $\varphi$,

$$
\begin{aligned}
\|\hat{e}\|_{L_{2}^{\psi^{-1}(\Omega)}}^{2}= & \sum_{n=0}^{N-1}\left(u_{h, t}+f_{R}\left(u_{h}\right)_{x}, \Psi-\varphi\right)_{n}+\sum_{n=0}^{N-1}\left(\hat{\varepsilon} u_{h, x},(\Psi-\varphi)_{x}\right)_{n} \\
& +\sum_{n=0}^{N-1}\left\langle\left[u_{h}\right],(\Psi-\varphi)_{+}\right\rangle_{n} .
\end{aligned}
$$

To proceed we use the following interpolation estimates, proofs of which can be found in [13] and [15]: 
Lemma 3.1 There is a constant $C_{i}$ such that for $\mathcal{R} \in L_{2}(\Omega)$

$$
\begin{aligned}
&\left|(\mathcal{R}, \varphi-\mathcal{P} \varphi)_{\Omega}\right|\left.\leq C_{i} \| \frac{h^{2}}{\hat{\varepsilon}}(\mathbb{I}-\mathcal{P}) \mathcal{R}\right)\left\|_{L_{2}^{\psi^{-1}}(\Omega)}\right\| \hat{\varepsilon} \varphi_{x x} \|_{L_{2}^{\psi}(\Omega)}, \\
&\left|\left(\hat{\varepsilon} u_{h, x},(\varphi-\mathcal{P} \varphi)_{x}\right)_{\Omega}\right| \leq C_{i}\left\|h^{2} \mathcal{R}_{1}\right\|_{L_{2}^{\psi-1}(\Omega)}\left\|\hat{\varepsilon} \varphi_{x x}\right\|_{L_{2}^{\psi}(\Omega)} .
\end{aligned}
$$

Similarly we have also the following Lemma, proof of which can be found in [11].

Lemma 3.2 There is a constant $C_{i}$ such that for $\mathcal{R} \in L_{2}^{\psi^{-1}}(\Omega)$

$$
\left|(\mathcal{R}, \mathcal{P}(\pi \varphi-\varphi))_{\Omega}\right| \leq C_{i}\left\|k_{n} \mathcal{R}\right\|_{L_{2}^{\psi^{-1}}(\Omega)}\left\|\varphi_{t}\right\|_{L_{2}^{\psi}(\Omega)} .
$$

We introduce the stability factors $S_{\hat{e}}^{t}$ and $S_{\hat{e}}^{x}$ associated with discretization in time and space, defined by

$$
S_{\hat{e}}^{t}=\frac{\left\|\varphi_{t}\right\|_{L_{2}^{\psi}(\Omega)}}{\|\hat{e}\|_{L_{2}^{\psi^{-1}}(\Omega)}}, \quad S_{\hat{e}}^{x}=\frac{\left\|\hat{\varepsilon} \varphi_{x x}\right\|_{L_{2}^{\psi}(\Omega)}}{\|\hat{e}\|_{L_{2}^{\psi^{-1}}}(\Omega)},
$$

respectively. Using Cauchy-Schwarz inequality in (3.4) coupled with the interpolation estimates (3.5a), (3.5b) and (3.6) and the strong stability factors (3.7), to derive the $L_{2}\left(L_{2}\right)$ a posteriori error estimates for the scheme (2.4).

Theorem 3.1 The error $\hat{e}=\hat{u}-u_{h}$, where $\hat{u}$ is the solution of the perturbed problem and $u_{h}$ that of (2.4) satisfies

$$
\begin{aligned}
\left\|\hat{u}-u_{h}\right\|_{L_{2}^{\psi-1}(\Omega)} \leq & \left.C_{i} S_{\hat{e}}^{x} \| \frac{h^{2}}{\hat{\varepsilon}}(\mathbb{I}-\mathcal{P}) \mathcal{R}_{0}\right)\left\|_{L_{2}^{\psi^{-1}}(\Omega)}+C_{i} S_{\hat{e}}^{t}\right\| k_{n} \mathcal{R}_{0} \|_{L_{2}^{\psi^{-1}}(\Omega)} \\
& +C_{i} S_{\hat{e}}^{x}\left\|h^{2} \mathcal{R}_{1}\right\|_{L_{2}^{\psi^{-1}}(\Omega)}+C_{i} S_{\hat{e}}^{t}\left\|k_{n} \mathcal{R}_{2}\right\|_{L_{2}^{\psi^{-1}}(\Omega)} \\
& +C_{i} S_{\hat{e}}^{x}\left\|\frac{h^{2}}{\hat{\varepsilon}} \mathcal{R}_{3}\right\|_{L_{2}^{\psi^{-1}}(\Omega)}+C_{i} S_{\hat{e}}^{t}\left\|k_{n} \mathcal{R}_{3}\right\|_{L_{2}^{\psi^{-1}}(\Omega)} \\
& +C_{i} S_{\hat{e}}^{t}\left\|k_{n} \mathcal{R}_{4}\right\|_{L_{2}^{\psi^{-1}}(\Omega)}
\end{aligned}
$$

Proof Using the notation introduced above, we may write (3.4) as

$$
\begin{aligned}
\|\hat{e}\|_{L_{2}^{\psi^{-1}(\Omega)}}^{2}= & \sum_{n=0}^{N-1}\left(\mathcal{R}_{0}, \Psi-\varphi\right)_{n}+\sum_{n=0}^{N-1}\left(\hat{\varepsilon} u_{h, x},(\Psi-\varphi)_{x}\right)_{n} \\
& +\sum_{n=0}^{N-1}\left\langle\left[u_{h}\right],(\Psi-\varphi)_{+}\right\rangle_{n} \equiv I+I I+I I I .
\end{aligned}
$$


Writing $\Psi-\varphi=\Psi-\mathcal{P} \varphi+\mathcal{P} \varphi-\varphi$ we obtain

$$
\begin{aligned}
I & =\sum_{n=0}^{N-1}\left(\mathcal{R}_{0}, \Psi-\mathcal{P} \varphi+\mathcal{P} \varphi-\varphi\right)_{n}=\sum_{n=0}^{N-1}\left(\mathcal{R}_{0}, \mathcal{P} \varphi-\phi\right)_{n}+\sum_{n=0}^{N-1}\left(\mathcal{R}_{0}, \mathcal{P}(\pi \varphi-\varphi)\right)_{n} \\
& \left.\leq C_{i} \| \frac{h^{2}}{\hat{\varepsilon}}(\mathbb{I}-\mathcal{P}) \mathcal{R}_{0}\right)\left\|_{L_{2}^{\psi-1}(\Omega)}\right\| \hat{\varepsilon} \varphi_{x x}\left\|_{L_{2}^{\psi}(\Omega)}+\right\| k_{n} \mathcal{R}_{0}\left\|_{L_{2}^{\psi-1}}\right\| \varphi_{(\Omega)} \|_{L_{2}^{\psi}(\Omega)},
\end{aligned}
$$

where we have used (3.5a) in the first integral and (3.6) in the second integral. For the term $I I$, we get the following bound, using (3.5b) and the fact that $D_{2}^{\hat{\varepsilon}}$ is constant on $I_{n}$,

$$
\begin{aligned}
I I & =\sum_{n=0}^{N-1}\left(\hat{\varepsilon} u_{h, x},(\Psi-\mathcal{P} \varphi+\mathcal{P} \varphi-\varphi)_{x}\right)_{n} \\
& =\sum_{n=0}^{N-1}\left(\hat{\varepsilon} u_{h, x},(\mathcal{P} \varphi-\varphi)_{x}\right)_{n}+\sum_{n=0}^{N-1}\left(\hat{\varepsilon} u_{h, x},(\Psi-\mathcal{P} \varphi)_{x}\right)_{n} \\
& =\sum_{n=0}^{N-1}\left(\hat{\varepsilon} u_{h, x},(\mathcal{P} \varphi-\varphi)_{x}\right)_{n}+\sum_{n=0}^{N-1}\left(-D_{2}^{\varepsilon} u_{h}, \mathcal{P}(\pi \varphi-\varphi)\right)_{n} \\
\leq & C_{i}\left(\left\|h^{2} \mathcal{R}_{1}\right\|_{L_{2}^{\psi^{-1}}(\Omega)}\left\|\hat{\varepsilon} \varphi_{x x}\right\|_{L_{2}^{\psi}(\Omega)}+\left\|k_{n} \mathcal{R}_{2}\right\|_{L_{2}^{\psi-1}(\Omega)}\left\|\varphi_{t}\right\|_{L_{2}^{\psi}(\Omega)}\right) .
\end{aligned}
$$

Finally, for the third term III in the error representation we have

$$
\begin{aligned}
I I I & =\sum_{n=0}^{N-1}\left\langle\left[u_{h}\right],(\Psi-\varphi)_{+}\right\rangle_{n} \\
& =\sum_{n=0}^{N-1}\left\langle\left[u_{h}\right],(\mathcal{P} \varphi-\varphi)_{+}\right\rangle_{n}+\sum_{n=0}^{N-1}\left\langle\left[u_{h}\right],(\pi \mathcal{P} \varphi-\mathcal{P} \varphi)_{+}\right\rangle_{n} \\
& \equiv I I I_{1}+I I I_{2} .
\end{aligned}
$$

Considering first $I I I_{1}$, we have with the $L_{2}$-projection $\mathcal{P}_{n}$ defined above

$$
\begin{aligned}
I I I_{1} & =\sum_{n=0}^{N-1}\left\langle u_{h,+}-u_{h,-},\left(\mathcal{P}_{n}-\mathbb{I}\right) \varphi_{+}\right\rangle_{n} \\
& =\sum_{n=0}^{N-1}\left\langle\mathcal{P}_{n} u_{h,-}-u_{h,-},\left(\mathbb{I}-\mathcal{P}_{n}\right)\left(\mathcal{P}_{n}-\mathbb{I}\right) \varphi_{+}\right\rangle_{n} \\
& =\sum_{n=0}^{N-1}\left\langle\mathcal{R}_{3}, k_{n}\left(\mathcal{P}_{n}-\mathbb{I}\right) \varphi_{+}\right\rangle_{n} .
\end{aligned}
$$


Now to estimate $\left(\mathbb{I}-\mathcal{P}_{n}\right) \varphi_{+}^{n}$ we note that

$$
k_{n} \varphi_{+}^{n}(x)=\int_{I_{n}} \varphi(x, t)-\int_{I_{n}} \int_{t_{n}}^{t} \varphi_{\tau}(x, \tau) d \tau d t .
$$

Inserting this representation into the right hand side of (3.10), using an estimate for $\left(\mathcal{P}_{n}-\mathbb{I}\right)$ we get

$$
\begin{aligned}
I I I_{1} & =\sum_{n=0}^{N-1}\left\langle\mathcal{R}_{3},\left(\mathcal{P}_{n}-\mathbb{I}\right)\left(\int_{I_{n}} \varphi(x, t) d t-\int_{I_{n}} \int_{t_{n}}^{t} \varphi_{\tau}(x, \tau) d \tau d t\right)\right\rangle_{n} \\
& =\sum_{n=0}^{N-1} \int_{I_{n}}\left\{\left\langle\mathcal{R}_{3},\left(\mathcal{P}_{n}-\mathbb{I}\right) \varphi\right\rangle_{n}-\int_{t_{n}}^{t}\left\langle\mathcal{R}_{3},\left(\mathcal{P}_{n}-\mathbb{I}\right) \varphi_{\tau}(\cdot, \tau)\right\rangle_{n} d \tau\right\} d t \\
& \leq C\left(\left\|\frac{h^{2}}{\hat{\varepsilon}} \mathcal{R}_{3}\right\|_{L_{2}^{\psi^{-1}}(\Omega)}\left\|\hat{\varepsilon} \varphi_{x x}\right\|_{L_{2}^{\psi}(\Omega)}+\left\|k_{n} \mathcal{R}_{3}\right\|_{L_{2}^{\psi^{-1}}(\Omega)}\left\|\varphi_{t}\right\|_{L_{2}^{\psi}(\Omega)}\right),
\end{aligned}
$$

where in the last inequality we have used that

$$
\left\|\mathcal{P}_{n} \varphi_{t}\right\|_{L_{2}^{\psi}(\Omega)} \leq\left\|\varphi_{t}\right\|_{L_{2}^{\psi}(\Omega)} .
$$

Finally, for $\mathrm{III}_{2}$ we have

$$
\begin{aligned}
I I_{2} & =\sum_{n=0}^{N-1}\left\langle k_{n} \frac{\left[u_{h}\right]}{k_{n}},(\pi \mathcal{P} \varphi-\mathcal{P} \varphi)_{+}\right\rangle_{n}=\sum_{n=0}^{N-1}\left\langle k_{n} \mathcal{R}_{4},(\pi \mathcal{P} \varphi-\mathcal{P} \varphi)_{+}\right\rangle_{n} \\
& =\sum_{n=0}^{N-1}\left\langle\mathcal{R}_{4},(\pi \mathcal{P}-\mathcal{P})\left(\int_{I_{n}} \varphi(x, t)-\int_{I_{n}} \int_{t_{n}}^{t} \varphi_{\tau}(x, \tau) d \tau d t\right)\right\rangle_{n} \\
& \leq C\left\|k_{n} \mathcal{R}_{4}\right\|_{L_{2}^{\psi^{-1}}(\Omega)}\left\|\varphi_{t}\right\|_{L_{2}^{\psi}(\Omega)} .
\end{aligned}
$$

The a posteriori error estimate now follows immediately after collecting the terms and using the definition of the stability factors (3.7).

\subsection{An a posteriori error estimates in $L_{\infty}\left(H^{-1}\right)$}

In principle, we may seek to control the error in any given norm by comparing the corresponding stability factors by solving a dual problem with suitable data. The norm $L_{\infty}\left(H^{-1}\right)$ offers certain advantages from analysis point of view, and connects to the following data for a dual linearized equation

$$
\begin{aligned}
\mathcal{L}_{\hat{\varepsilon}}^{*} \varphi & =0 \quad \text { in } \Omega, \\
\varphi(0, t) & =0, \quad t \in(0, T), \\
\varphi(x, T) & =E, \quad x \in \mathbb{R}_{+},
\end{aligned}
$$


where

$$
-E_{x x}=\hat{e}, \quad x \in \mathbb{R}_{+}, \quad|E| \rightarrow 0 \quad \text { as }|x| \rightarrow \infty .
$$

We define the norm $\|\hat{e}\|_{H^{-1}}$ by

$$
\|\hat{e}\|_{H^{-1}}=\left(E_{x}(T), E_{x}(T)\right)_{\mathbb{R}_{+}},
$$

and note the following error representation based on (3.13)

$$
\begin{aligned}
\|\hat{e}\|_{H^{-1}}^{2}= & \left\|E_{x}\right\|_{L_{2}}^{2}=\left\langle\hat{e}_{-}, \varphi\right\rangle_{N}-\sum_{n=0}^{N-1}\left(\hat{e}, \mathcal{L}_{\hat{\varepsilon}}^{*} \varphi\right)_{n} \\
= & \left\langle\hat{e}_{-}, \varphi\right\rangle_{N}-\left\langle\hat{e}_{-}, \varphi\right\rangle_{N}+\langle\hat{e}, \varphi\rangle_{0}+\sum_{n=0}^{N-1}\left(\mathcal{L}_{\hat{\varepsilon}} u, \varphi\right)_{n} \\
& -\sum_{n=0}^{N-1}\left\{\left(u_{h, t}+f_{R}\left(u_{h}\right)_{x}, \varphi\right)_{n}-\left(\hat{\varepsilon} u_{h, x}, \varphi_{x}\right)_{n}\right\}-\sum_{n=0}^{N-1}\left\langle\left[u_{h}\right], \varphi_{+}\right\rangle_{n} \\
= & \langle\hat{e}, \varphi\rangle_{0}-\sum_{n=0}^{N-1}\left\{\left(u_{h, t}+f_{R}\left(u_{h}\right)_{x}, \varphi\right)_{n}-\left(\hat{\varepsilon} u_{h, x}, \varphi_{x}\right)_{n}\right\}-\sum_{n=0}^{N-1}\left\langle\left[u_{h}\right], \varphi_{+}\right\rangle_{n} .
\end{aligned}
$$

Using Galerkin orthogonality for $\Psi \in \mathcal{V}_{h}$ and with $\langle\hat{e}, \varphi\rangle_{0}=0$ we have

$$
\begin{aligned}
\|\hat{e}\|_{H^{-1}}^{2}= & \sum_{n=0}^{N-1}\left(u_{h, t}+f_{R}\left(u_{h}\right)_{x}, \Psi-\varphi\right)_{n}-\sum_{n=0}^{N-1}\left(\hat{\varepsilon} u_{h, x}, \Psi_{x}-\varphi_{x}\right)_{n} \\
& +\sum_{n=0}^{N-1}\left\langle\left[u_{h}\right],(\Psi-\varphi)_{+}\right\rangle_{n} .
\end{aligned}
$$

We introduce the following stability factors associated with discretization in time and space respectively,

$$
S_{E}^{t}=\frac{\left\|\varphi_{t}\right\|_{L_{1}\left(L_{2}\left(\mathbb{R}_{+}\right)\right)}}{\|\hat{e}\|_{H^{-1}}}, \quad S_{E}^{x}=\frac{\left\|\sqrt{\hat{\varepsilon}} \varphi_{x x}\right\|_{L_{1}\left(L_{2}\left(\mathbb{R}_{+}\right)\right)}}{\|\hat{e}\|_{H^{-1}}}
$$

Using interpolation estimates of the same type as those in the previous section we obtain the following a posteriori error estimate.

Theorem 3.2 The error $\hat{e}=\hat{u}-u_{h}$, where $\hat{u}$ is the solution of the perturbed problem and $u_{h}$ that of (2.4) satisfies

$$
\left.\|\hat{e}\|_{H^{-1}} \leq C_{i} S_{E}^{x} \| \frac{h^{2}}{\sqrt{\hat{\varepsilon}}}(\mathbb{I}-\mathcal{P}) \mathcal{R}_{0}\right)\left\|_{L_{\infty}\left(L_{2}\left(\mathbb{R}_{+}\right)\right)}+C_{i} S_{E}^{t}\right\| k_{n} \mathcal{R}_{0} \|_{L_{\infty}\left(L_{2}\left(\mathbb{R}_{+}\right)\right)}
$$




$$
\begin{aligned}
& +C_{i} S_{E}^{x}\left\|h^{2} \mathcal{R}_{1}\right\|_{L_{\infty}\left(L_{2}\left(\mathbb{R}_{+}\right)\right)}+C_{i} S_{E}^{t}\left\|k_{n} \mathcal{R}_{2}\right\|_{L_{\infty}\left(L_{2}\left(\mathbb{R}_{+}\right)\right)} \\
& +C_{i} S_{E}^{x}\left\|\frac{h^{2}}{\sqrt{\hat{\varepsilon}}} \mathcal{R}_{3}\right\|_{L_{\infty}\left(L_{2}\left(\mathbb{R}_{+}\right)\right)}+C_{i} S_{E}^{t}\left\|k_{n} \mathcal{R}_{3}\right\|_{L_{\infty}\left(L_{2}\left(\mathbb{R}_{+}\right)\right)} \\
& +C_{i} S_{E}^{t}\left\|k_{n} \mathcal{R}_{4}\right\|_{L_{\infty}\left(L_{2}\left(\mathbb{R}_{+}\right)\right)} .
\end{aligned}
$$

The proof is essentially the same as the proof of the $L_{2}(\Omega)$ case and is therefore omitted. We notice a gain of a factor $\sqrt{\hat{\varepsilon}}$ as compared to (3.8) due to the asymmetry of norms in (3.16), with the weaker $H^{-1}\left(\mathbb{R}_{+}\right)$-norm on the left hand side.

\section{Analytical strong stability estimates}

To give the above a posteriori estimate a quantitative meaning we need to estimate the strong stability factors. We present some stability estimates for the model problem indicating that the corresponding stability factor is bounded by a moderate constant. More specifically, to get a quantitative estimate of the computational error $\left\|u-u_{h}\right\|$ in terms of $S_{\hat{e}}^{t}, S_{E}^{t}, S_{\hat{e}}^{x}$ and $S_{E}^{x}$, it is necessary that these two quantities are bounded by some moderate constants.

\subsection{Strong stability in $L_{2}(\Omega)$}

In this section we consider the a posteriori error estimate of the type (3.8) derived in the previous section based on the following dual problem

$$
\begin{aligned}
\mathcal{L}_{\hat{\varepsilon}}^{*} \varphi \equiv-\varphi_{t}-A^{T} \varphi_{x}-\hat{\varepsilon} \varphi_{x x} & =\psi^{-1} \hat{e} \quad \text { in } \Omega, \\
\varphi(0, t) & =0, \quad t \in(0, T), \\
\varphi(x, T) & =0, \quad x \in \mathbb{R}_{+},
\end{aligned}
$$

where we assume that $\varphi(x, t) \rightarrow 0$ as $x \rightarrow \infty$, for all $t$. We now prove the following strong stability estimate for the dual problem (4.1).

Theorem 4.1 Let $\hat{\varepsilon}>0, \alpha \geq 0$ and $\beta \geq 0$ be constants. Suppose further that $\psi(x, t)$ is a positive weight function satisfying

$$
\psi_{t}+A^{T} \psi_{x}-A_{x}^{T} \psi-2 \hat{\varepsilon} \psi_{x}^{2} \psi^{-1} \geq \alpha\left|A_{x}^{T}\right| \psi+\beta \psi \quad \text { in } \Omega
$$

then the solution $\varphi$ of (4.1) satisfies

$$
\begin{gathered}
\left\|\varphi_{t}+A^{T} \varphi_{x}\right\|_{L_{2}^{\psi}(\Omega)}^{2}+\left\|\hat{\varepsilon} \varphi_{x x}\right\|_{L_{2}^{\psi}(\Omega)}^{2}+\left\|\hat{\varepsilon}^{\frac{1}{2}} \varphi_{x}\right\|_{L_{\infty}\left(L_{2}^{\psi}(\Omega)\right)}^{2} \\
+2\left\|\hat{\varepsilon}^{\frac{1}{2}}\left(\alpha\left|A_{x}^{T}\right|+\beta\right)^{\frac{1}{2}} \varphi_{x}\right\|_{L_{2}^{\psi}(\Omega)}^{2} \leq 6\|\hat{e}\|_{L_{2}^{\psi-1}(\Omega)}^{2},
\end{gathered}
$$


and

$$
\left\|\varphi_{t}\right\|_{L_{2}^{\psi}(\Omega)}^{2} \leq 4\left(3+T \sup _{\Omega}\left\|\frac{A^{T} A}{\hat{\varepsilon}}\right\|\right)\|\hat{e}\|_{L_{2}^{\psi^{-1}}(\Omega)}^{2} .
$$

Proof We multiply (4.1a) by $-\psi\left(\varphi_{t}+A^{T} \varphi_{x}\right)$ and integrate over $\Omega_{\tau}=\mathbb{R}_{+} \times(\tau, T)$ to get

$$
\begin{gathered}
\left\|\psi^{\frac{1}{2}}\left(\varphi_{t}+A^{T} \varphi_{x}\right)\right\|_{\Omega_{\tau}}^{2}+\int_{\Omega_{\tau}} \psi\left(\varphi_{t}+A^{T} \varphi_{x}\right) \hat{\varepsilon} \varphi_{x x} d x d t \\
=-\int_{\Omega_{\tau}} \hat{e}\left(\varphi_{t}+A^{T} \varphi_{x}\right) d x d t \\
\quad \leq \int_{\Omega_{\tau}} \psi^{-1} \hat{e}^{2} d x d t+\frac{1}{4}\left\|\psi^{1 / 2}\left(\varphi_{t}+A^{T} \varphi_{x}\right)\right\|_{\Omega_{\tau}}^{2} .
\end{gathered}
$$

Integrating by parts in both space and time variables in the second term on the lefthand side yields

$$
\begin{aligned}
\int_{\Omega_{\tau}} \psi\left(\varphi_{t}+A^{T} \varphi_{x}\right) \hat{\varepsilon} \varphi_{x x} d x d t \\
=\int_{\Omega_{\tau}} \psi \varphi_{t} \hat{\varepsilon} \varphi_{x x}+\int_{\Omega_{\tau}} \psi A^{T} \varphi_{x} \hat{\varepsilon} \varphi_{x x} d x d t \\
=-\int_{\Omega_{\tau}} \hat{\varepsilon} \psi_{x} \varphi_{x} \varphi_{t} d x d t-\int_{\Omega_{\tau}} \hat{\varepsilon} \psi \varphi_{x} \varphi_{t x} d x d t \\
-\frac{1}{2} \int_{\Omega_{\tau}} \hat{\varepsilon} \psi A_{x}^{T} \varphi_{x}^{2} d x d t-\frac{1}{2} \int_{\Omega_{\tau}} \hat{\varepsilon} \psi_{x} A^{T} \varphi_{x}^{2} d x d t \\
=-\int_{\Omega_{\tau}} \hat{\varepsilon} \psi_{x}\left(\varphi_{t}+A^{T} \varphi_{x}\right) \varphi_{x} d x d t-\frac{1}{2} \int_{\Omega_{\tau}}\left(\hat{\varepsilon} \psi \varphi_{x}^{2}\right)_{t} d x d t \\
+\frac{1}{2} \int_{\Omega_{\tau}} \hat{\varepsilon} \psi_{t} \varphi_{x}^{2} d x d t+\frac{1}{2} \int_{\Omega_{\tau}} \hat{\varepsilon} \varphi_{x}^{2}\left(A^{T} \psi_{x}-A_{x}^{T} \psi\right) d x d t
\end{aligned}
$$

By substituting into above inequality we have

$$
\begin{aligned}
& \frac{3}{4}\left\|\psi^{\frac{1}{2}}\left(\varphi_{t}+A^{T} \varphi_{x}\right)\right\|_{\Omega_{\tau}}^{2}+\frac{1}{2} \int_{\mathbb{R}_{+}}\left(\hat{\varepsilon} \psi \varphi_{x}^{2}\right)(x, \tau) d x \\
& \quad+\frac{1}{2} \int_{\Omega_{\tau}} \hat{\varepsilon} \varphi_{x}^{2}\left(\psi_{t}+A^{T} \psi_{x}-A_{x}^{T} \psi\right) d x d t \\
& \leq\left\|\psi^{-\frac{1}{2}} \hat{e}\right\|_{\Omega_{\tau}}^{2}+\int_{\Omega_{\tau}} \hat{\varepsilon} \psi_{x} \varphi_{x}\left(\varphi_{t}+A^{T} \varphi_{x}\right) d x d t \\
& \leq\left\|\psi^{-\frac{1}{2}} \hat{e}\right\|_{\Omega_{\tau}}^{2}+\int_{\Omega_{\tau}} \hat{\varepsilon}^{2} \psi_{x}^{2} \varphi_{x}^{2} \psi^{-1} d x d t+\frac{1}{4}\left\|\psi^{\frac{1}{2}}\left(\varphi_{t}+A^{T} \varphi_{x}\right)\right\|_{\Omega_{\tau}}^{2} .
\end{aligned}
$$


Collecting terms, we obtain that

$$
\begin{aligned}
& \left\|\psi^{\frac{1}{2}}\left(\varphi_{t}+A^{T} \varphi_{x}\right)\right\|_{\Omega_{\tau}}^{2}+\int_{\mathbb{R}_{+}}\left(\hat{\varepsilon} \psi \varphi_{x}^{2}\right)(x, \tau) d x \\
& \quad+\int_{\Omega_{\tau}} \hat{\varepsilon} \varphi_{x}^{2}\left(\psi_{t}+A^{T} \psi_{x}-A_{x}^{T} \psi-2 \hat{\varepsilon} \psi_{x}^{2} \psi^{-1}\right) d x d t \leq 2\left\|\psi^{-\frac{1}{2}} \hat{e}\right\|_{\Omega_{\tau}}^{2} .
\end{aligned}
$$

We may now choose $\tau$ such that

$$
\int_{\mathbb{R}_{+}}\left(\hat{\varepsilon} \psi \varphi_{x}^{2}\right)(x, \tau) d x=\sup _{t \in[0, T]} \int_{\mathbb{R}_{+}}\left(\hat{\varepsilon} \psi \varphi_{x}^{2}\right)(\cdot, t) d x=\left\|\hat{\varepsilon}^{\frac{1}{2}} \varphi_{x}\right\|_{L_{\infty}\left(L_{2}^{\psi}\left(\Omega_{\tau}\right)\right)}^{2},
$$

and use (4.2) to obtain

$$
\begin{aligned}
& \left\|\hat{\varepsilon}^{\frac{1}{2}} \varphi_{x}\right\|_{L_{\infty}\left(L_{2}^{\psi}\left(\Omega_{\tau}\right)\right)}^{2}+\left\|\hat{\varepsilon}^{\frac{1}{2}}\left(\alpha\left|A_{x}^{T}\right|+\beta\right)^{\frac{1}{2}} \varphi_{x}\right\|_{\Omega_{\tau}}^{2} \\
& \quad \leq 2\left\|\psi^{-\frac{1}{2}} \hat{e}\right\|_{\Omega_{\tau}}^{2} \leq 2\left\|\psi^{-\frac{1}{2}} \hat{e}\right\|_{\Omega}^{2} .
\end{aligned}
$$

Choosing $\tau=0$ yields the following inequality

$$
\left\|\psi^{\frac{1}{2}}\left(\varphi_{t}+A^{T} \varphi_{x}\right)\right\|_{\Omega}^{2}+\left\|\hat{\varepsilon}^{\frac{1}{2}}\left(\alpha\left|A_{x}^{T}\right|+\beta\right)^{\frac{1}{2}} \varphi_{x} \psi^{\frac{1}{2}}\right\|_{\Omega}^{2} \leq 2\left\|\psi^{-\frac{1}{2}} \hat{e}\right\|_{\Omega}^{2} .
$$

Similarly, to obtain a bound for $\left\|\hat{\varepsilon} \psi^{\frac{1}{2}} \varphi_{x x}\right\|_{\Omega}$, multiply equation (4.1a) by $-\hat{\varepsilon} \psi \varphi_{x x}$ and integrating over $\Omega_{\tau}$ to get

$$
\begin{aligned}
& \left\|\hat{\varepsilon} \psi^{\frac{1}{2}} \varphi_{x x}\right\|_{\Omega_{\tau}}^{2}+\int_{\Omega_{\tau}} \hat{\varepsilon} \psi \varphi_{x x}\left(\varphi_{t}+A^{T} \varphi_{x}\right) d x d t \\
& =-\int_{\Omega_{\tau}} \hat{\varepsilon} \varphi_{x x} \hat{e} \leq\left\|\psi^{-\frac{1}{2}} \hat{e}\right\|_{\Omega_{\tau}}^{2}+\frac{1}{4}\left\|\hat{\varepsilon} \psi^{\frac{1}{2}} \varphi_{x x}\right\|_{\Omega_{\tau}}^{2} .
\end{aligned}
$$

Using the integration by part for the second term in the left-hand side as in (4.5) and also using (4.2) we obtain

$$
\left\|\hat{\varepsilon} \psi^{\frac{1}{2}} \varphi_{x x}\right\|_{\Omega}^{2}+\left\|\hat{\varepsilon}^{\frac{1}{2}} \psi^{\frac{1}{2}}\left(\alpha\left|A_{x}^{T}\right|+\beta\right)^{\frac{1}{2}} \varphi_{x}\right\|_{\Omega}^{2} \leq 2\left\|\psi^{-\frac{1}{2}} \hat{e}\right\|_{\Omega}^{2} .
$$

Adding estimates (4.6), (4.7), (4.8) we have proved (4.3).

To prove (4.4) we writing (4.1a) in the form

$$
-\varphi_{t}-A^{T} \varphi_{x}=\psi^{-1} \hat{e}+\hat{\varepsilon} \varphi_{x x} .
$$

Taking the square on both sides, multiplying by $\psi$ and integrating over $\Omega$ yields

$$
\begin{gathered}
\left\|\varphi_{t}\right\|_{L_{2}^{\psi}(\Omega)}^{2}+\left\|A^{T} \varphi_{x}\right\|_{L_{2}^{\psi}(\Omega)}^{2}+\int_{\Omega} 2 A^{T} \varphi_{x} \varphi_{t} \psi d x d t \\
=\|\hat{e}\|_{L_{2}^{\psi-1}(\Omega)}^{2}+\left\|\hat{\varepsilon} \varphi_{x x}\right\|_{L_{2}^{\psi}(\Omega)}^{2}+\int_{\Omega} 2 \hat{\varepsilon} \hat{e} \varphi_{x x} .
\end{gathered}
$$


Recalling (4.8) and using the arithmetic geometric mean value inequality in the right hand side we have

$$
\left\|\varphi_{t}\right\|_{L_{2}^{\psi}(\Omega)}^{2}+\left\|A^{T} \varphi_{x}\right\|_{L_{2}^{\psi}(\Omega)}^{2} \leq 6\|\hat{e}\|_{L_{2}^{\psi^{-1}(\Omega)}}^{2}+2\left\|A^{T} \varphi_{x}\right\|_{L_{2}^{\psi}(\Omega)}^{2}+\frac{1}{2}\left\|\varphi_{t}\right\|_{L_{2}^{\psi}(\Omega)}^{2} .
$$

Noting that

$$
\left\|A^{T} \varphi_{x}\right\|_{L_{2}^{\psi}(\Omega)}^{2}=\left\|\left(A^{T} A\right)^{1 / 2} \varphi_{x}\right\|_{L_{2}^{\psi}(\Omega)}^{2},
$$

and using (4.6) we have

$$
\begin{aligned}
\frac{1}{2}\left\|\varphi_{t}\right\|_{L_{2}^{\psi}(\Omega)}^{2} & \leq 6\|\hat{e}\|_{L_{2}^{\psi \psi^{-1}}(\Omega)}^{2}+\sup _{\Omega}\left\|\frac{A^{T} A}{\hat{\varepsilon}}\right\|\left\|\hat{\varepsilon}^{1 / 2} \varphi_{x}\right\|_{L_{2}^{\psi}(\Omega)}^{2} \\
& \leq 2\left(3+T \sup _{\Omega}\left\|\frac{A^{T} A}{\hat{\varepsilon}}\right\|\right)\|\hat{e}\|_{L_{2}^{\psi-1}(\Omega)}^{2},
\end{aligned}
$$

which proves the desired result.

Corollary 4.1 The assumption (4.2) of Theorem 4.1 is satisfied in the following cases:

(i) $\psi \equiv 1$ and $A_{x}^{T} \leq 0, \beta=0, \alpha=1$, corresponding to a shock,

(ii) $\psi(x, t)=\left(\frac{t}{T}\right)^{1+\alpha}, \max A_{x}^{T}(\cdot, t) \leq \frac{1}{t}, \beta=0, \alpha \geq 0$, corresponding to rarefaction wave $u(x, t)=\frac{x}{t}$, and

(iii) $\psi=\exp (3 \beta(t-T))$ and $\left|A_{x}^{T}\right| \leq \beta, \alpha=1$, corresponding to regular solution, see [13].

\subsection{Strong stability in $L_{\infty}\left(H^{-1}\right)$}

We now prove a strong stability estimate for the $L_{\infty}\left(H^{-1}\right)$ norm a posteriori error estimate (3.16) based on the following dual problem

$$
\begin{aligned}
-\varphi_{t}-A^{T} \varphi_{x}-\hat{\varepsilon} \varphi_{x x} & =0 \quad \text { in } \Omega, \\
\varphi(0, t) & =0, \quad t \in(0, T), \\
\varphi(x, T) & =E, \quad x \in \mathbb{R}_{+}, \\
-E_{x x} & =\hat{e}(T), \quad x \in \mathbb{R}_{+} .
\end{aligned}
$$

Theorem 4.2 The solution $\varphi$ of (4.9) satisfies

$$
\begin{aligned}
\left\|\sqrt{\hat{\varepsilon}} \varphi_{x x}\right\|_{L_{1}\left(L_{2}\left(\mathbb{R}_{+}\right)\right)} & \leq S_{E}^{x} \sqrt{T}\|\hat{e}(T)\|_{H^{-1}}, \\
\left\|\varphi_{t}\right\|_{L_{1}\left(L_{2}\left(\mathbb{R}_{+}\right)\right)} & \leq S_{E}^{t} T\|\hat{e}(T)\|_{H^{-1}},
\end{aligned}
$$

where $S_{E}^{x}=\frac{1}{\sqrt{2}}$ and $S_{E}^{t}=\sqrt{\sup _{\Omega}\left\|A A^{T}\right\|+\hat{\varepsilon}}$. 
Proof We start by writing (4.9) in the form

$$
-\varphi_{t}-\hat{\varepsilon} \varphi_{x x}=A^{T} \varphi_{x}
$$

Taking the square on both sides, multiplying by $\psi$ and integrating over $\Omega$ yields

$$
\int_{\Omega} \varphi_{t}^{2} d x d t+\int_{\Omega} \hat{\varepsilon}^{2} \varphi_{x x}^{2} d x d t+\int_{\Omega} 2 \hat{\varepsilon} \varphi_{x x} \varphi_{t} d x d t=\int_{\Omega}\left(A^{T} \varphi_{x}\right)^{2} d x d t .
$$

Using partial integration we find that

$$
\left\|\varphi_{t}\right\|_{\Omega}^{2}+\left\|\hat{\varepsilon} \varphi_{x x}\right\|_{\Omega}^{2}+\int_{\mathbb{R}_{+}} \hat{\varepsilon} \varphi_{x}^{2}(., 0) d x=\int_{\Omega}\left(A^{T} \varphi_{x}\right)^{2} d x d t+\left\|\sqrt{\hat{\varepsilon}} E_{x}\right\|_{L_{2}\left(\mathbb{R}_{+}\right)}^{2} .
$$

Nothing that

$$
\int_{\Omega}\left(A^{T} \varphi_{x}\right)^{2} d x d t \leq T \sup _{\Omega}\left\|A A^{T}\right\|\left\|E_{x}\right\|_{L_{2}\left(\mathbb{R}_{+}\right)}^{2},
$$

and using (4.12) we get

$$
\left\|\varphi_{t}\right\|_{\Omega}^{2}+\left\|\hat{\varepsilon} \varphi_{x x}\right\|_{\Omega}^{2} \leq\left(T \sup _{\Omega}\left\|A A^{T}\right\|+\hat{\varepsilon}\right)\left\|E_{x}\right\|_{L_{2}\left(\mathbb{R}_{+}\right)}^{2} .
$$

Similarly to prove (4.10a) multiply (4.9) by $-\varphi_{x x}$ as in the $L_{2}$-norm example to get

$$
\sup _{t \in[0, T]} \int_{\mathbb{R}_{+}} \hat{\varepsilon} \varphi_{x}^{2}(., t) d x+\left\|\sqrt{\hat{\varepsilon}} \varphi_{x x}\right\|_{\Omega_{\tau}}^{2} \leq \frac{1}{2}\left\|E_{x}\right\|_{L_{2}\left(\mathbb{R}_{+}\right)}^{2},
$$

and with $\tau=0$

$$
\left\|\sqrt{\hat{\varepsilon}} \varphi_{x x}\right\|_{\Omega}^{2} \leq \frac{1}{2}\left\|E_{x}\right\|_{L_{2}\left(\mathbb{R}_{+}\right)}^{2} .
$$

The theorem follows from inequalities (4.13) and (4.14), noting that

$$
\left(\int\|\varphi\|\right)^{2} \leq T \int\|\varphi\|^{2} d t
$$

\section{Numerical implementations}

In this section we discuss the computation of the solution of the linearized dual problem and present some numerical results. By putting $f_{\alpha}(u)=a_{\alpha} u(\alpha=L, R)$ in (1.1) we get the linear case of our model problem

$$
\begin{cases}u_{t}+a_{R} u_{x}-(\varepsilon u)_{x x}=0, & x>0, t>0, \\ u_{t}+a_{L} u_{x}-(\varepsilon u)_{x x}=0, & x<0, t>0, \\ u(0, t)=0, & t>0, \\ u(x, 0)=u_{0}(x), & x \in \mathbb{R} .\end{cases}
$$


The associated linearized dual problem takes the form

$$
\begin{cases}\varphi_{t}+a_{R} \varphi_{x}+(\varepsilon \varphi)_{x x}=0, & x>0, t>0, \\ \varphi_{t}+a_{L} \varphi_{x}+(\varepsilon \varphi)_{x x}=0, & x<0, t>0, \\ \varphi(0, t)=0, & t>0, \\ \varphi(x, T)=E, & x \in \mathbb{R},\end{cases}
$$

where $-E_{x x}=e$, corresponding to error control in the $L_{\infty}\left(H^{-1}\right)$ norm. We have solved the dual problem using the approximations of the error $e=u-u_{h}$, with $u$ the analytical solution and $u_{h}$ the finite element solution on a mesh of size $h$.

In [12], implementation of finite element method (Sd-method) for linear case are considered. This methodology can be used to solve the dual problem as well (with some minor modification). In all cases below we consider examples with the exact solutions and present computed approximation of $S_{E}^{x}$ and $S_{E}^{t}$ defined by (3.15) for a typical solution of coupling of two advection-diffusion equations corresponding to a posteriori error estimate in $L_{\infty}\left(H^{-1}\right)$ norm, together with plots of dual solutions.

\subsection{Test case 1}

We first consider the following problem

$$
\begin{cases}u_{t}-\frac{1}{2} u_{x}-(\varepsilon u)_{x x}=0, & x \in(0,1], \\ u_{t}+\frac{1}{2} u_{x}-(\varepsilon u)_{x x}=0, & x \in[-1,0),\end{cases}
$$

where $t \in[0, T]$ with the following continuous initial data

$$
u(x, 0)= \begin{cases}4 x(1+x), & -1 \leq x \leq 0 \\ 4 x(1-x), & 0 \leq x \leq 1 \\ 0, & |x| \geq 1\end{cases}
$$

where $T=0.15$ is the final time and we have used fixed mesh size $h=10^{-3}$ in the computation.

The approximate solution $u$ on a uniform mesh by $\mathrm{Sd}$-method with $\varepsilon=10^{-6}$, time step $k / h=0.25$ and for initial and final time levels, $t=0$ and $t=T$ are presented in Fig. 1. We next plot the component of dual solution $\varphi$ and the corresponding their second derivatives $\varphi_{x x}$ at the same time levels, but in reverse order $t=T$ and $t=0$ in Fig. 2 that shows how the error $e_{-}^{N}=-E_{x x}=-\varphi_{x x}(T)$ is distributed. Stability factors $S_{E}^{x}$ and $S_{E}^{t}$ for this $\varepsilon$ are represented versus time in Fig. 3. Then we take $\varepsilon=10^{-4}$ and plot the corresponding dual solution and stability factors in Figs. 4 and 5 respectively. Finally we plot the variation of stability factors as a function of viscosity for $10^{-6} \leq \varepsilon \leq 10^{-4}$ in Fig. 6 .

In this case, $S_{E}^{x}$ and $S_{E}^{t}$ turn out to be of moderate size, indicating computability of the corresponding solutions in $L_{\infty}\left(H^{-1}\right)$. 

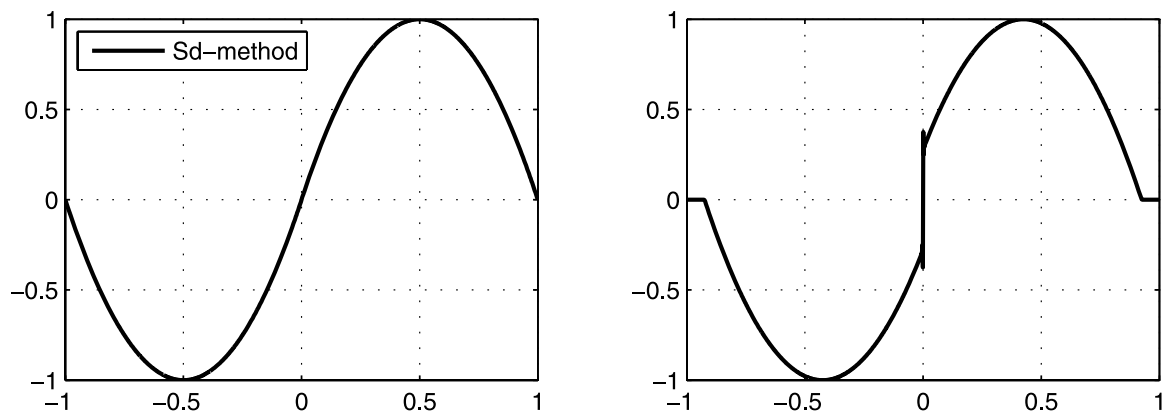

Fig. 1 Test case 1. Plot of $u$ by streamline diffusion method at $t=0$ (left) and $t=T$ (right) for $\varepsilon=10^{-6}$
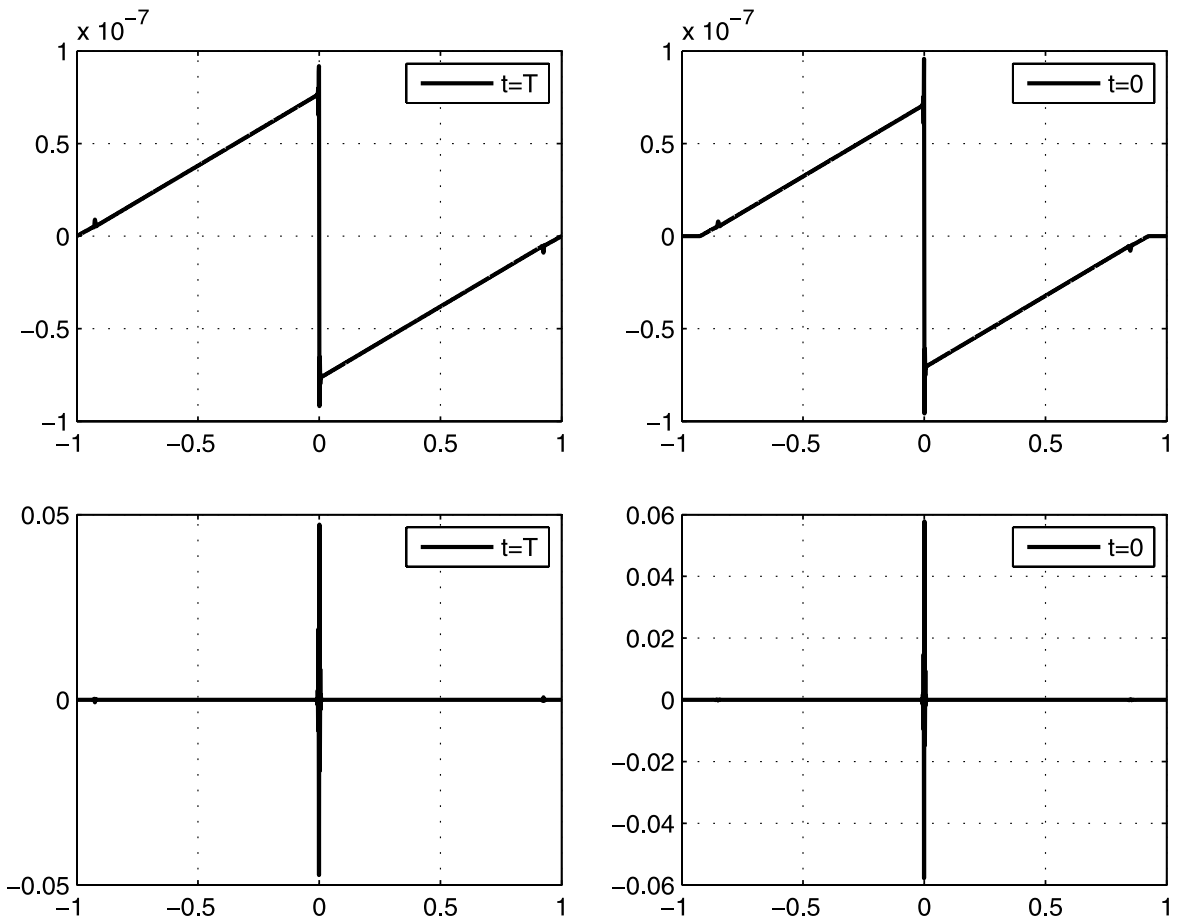

Fig. 2 Test case 1. Plot of $\varphi(t)$ (top) and $\varphi_{x x}(t)$ (bottom) at $t=T$ and $t=0$ for $\varepsilon=10^{-6}$

\subsection{Test case 2}

Finally we consider the above problem with the following initial condition with two discontinuities

$$
u(x, 0)= \begin{cases}1, & -1 \leq x \leq-0.25 \\ -1, & 0.25 \leq x \leq 1, \\ 0, & \text { otherwise }\end{cases}
$$



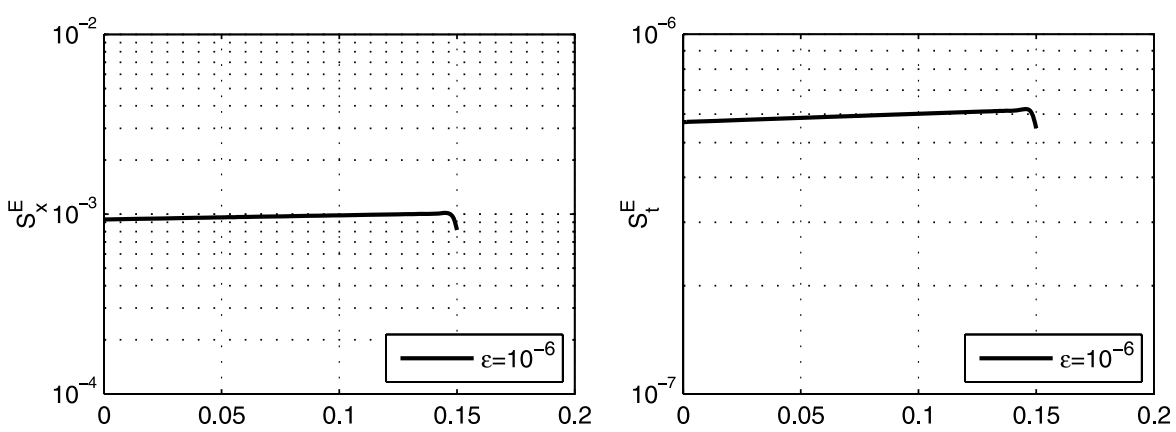

Fig. 3 Test case 1 . Plot of the stability factors $S_{E}^{x}$ and $S_{E}^{t}$ versus time for $\varepsilon=10^{-6}$
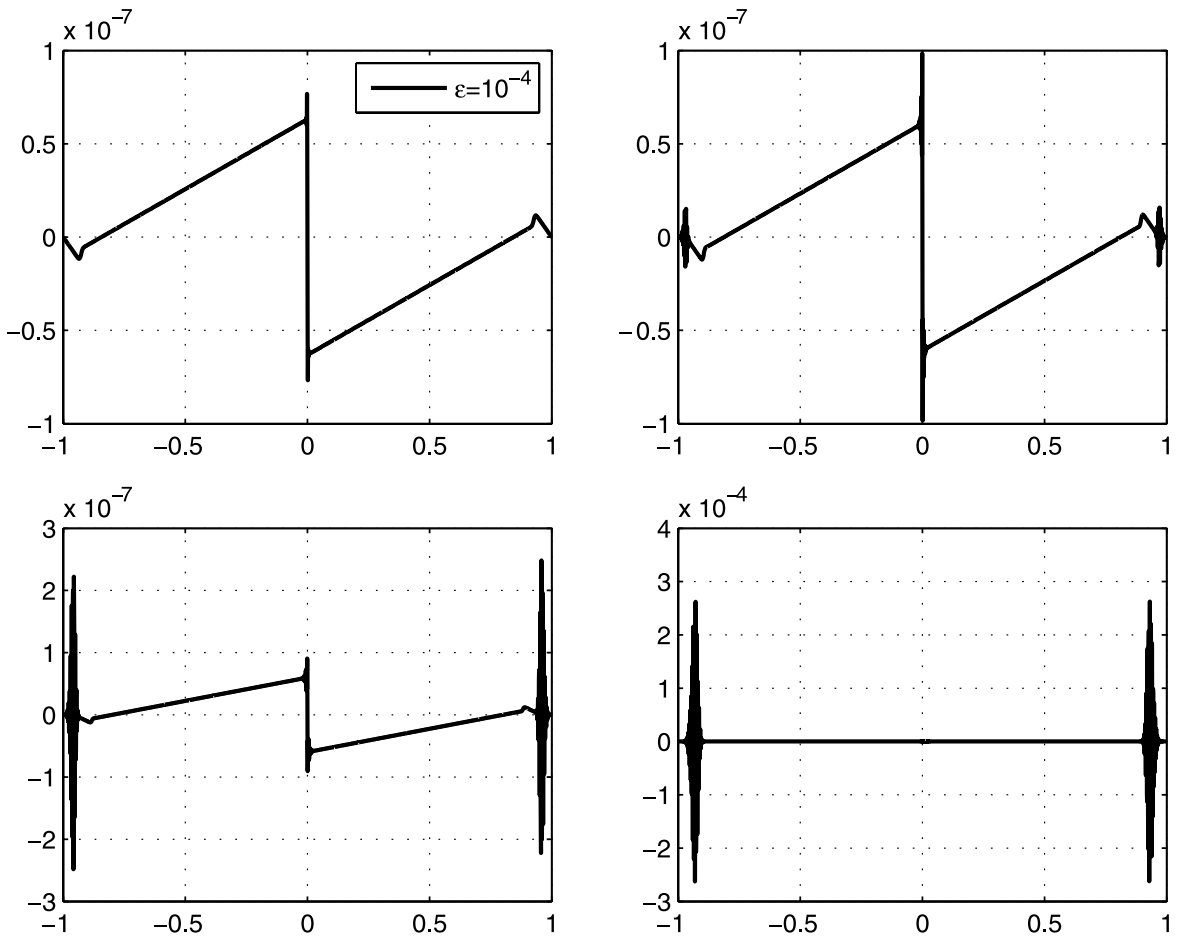

Fig. 4 Test case 1. Plot of the $\varphi(t)$ at $t=T, t=0.5 T$ (top), $t=0.4 T$ and $t=0$ (bottom) for $\varepsilon=10^{-4}$

with the parameters $a_{R}=-0.01, a_{L}=0.01$. Here we have used a fixed value on the viscosity, $\varepsilon=10^{-5}$. Firstly we solve the problem with $h=10^{-2}$ on a uniform mesh with time step $k / h=0.25$. We plot the computed solution $u$ by Sd-method at $t=0$ and $t=T$ in Fig. 7 and the corresponding dual solution $\varphi$, second their derivatives solutions $\varphi_{x x}$ at $t=T$ and $t=0$ in Fig. 8. We plot the stability factors corresponding to this $h$ in Fig. 9. Secondly we refine the mesh size to $h=10^{-3}$ and then plot the corresponding figures for dual solutions and their stability factors at $t=T, t=0.5 T$, 

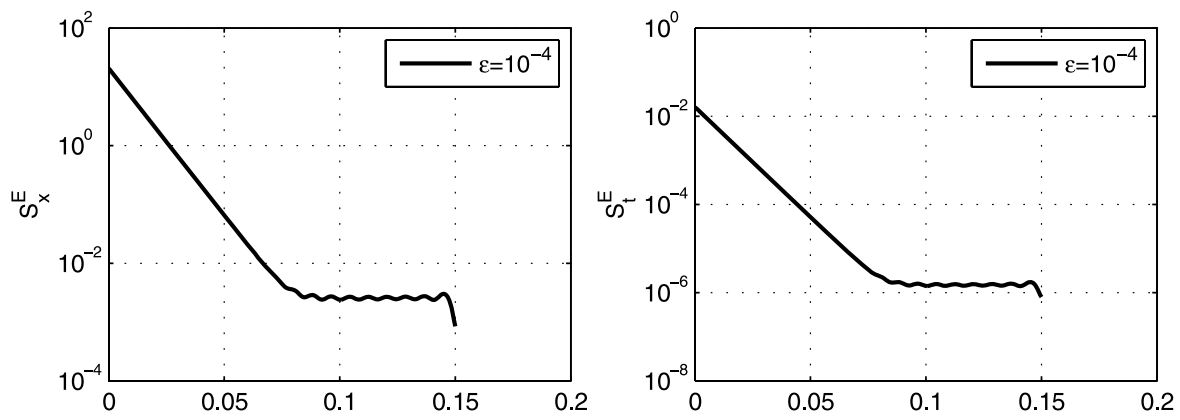

Fig. 5 Test case 1 . Plot of the stability factors $S_{E}^{x}$ and $S_{E}^{t}$ versus time for $\varepsilon=10^{-4}$

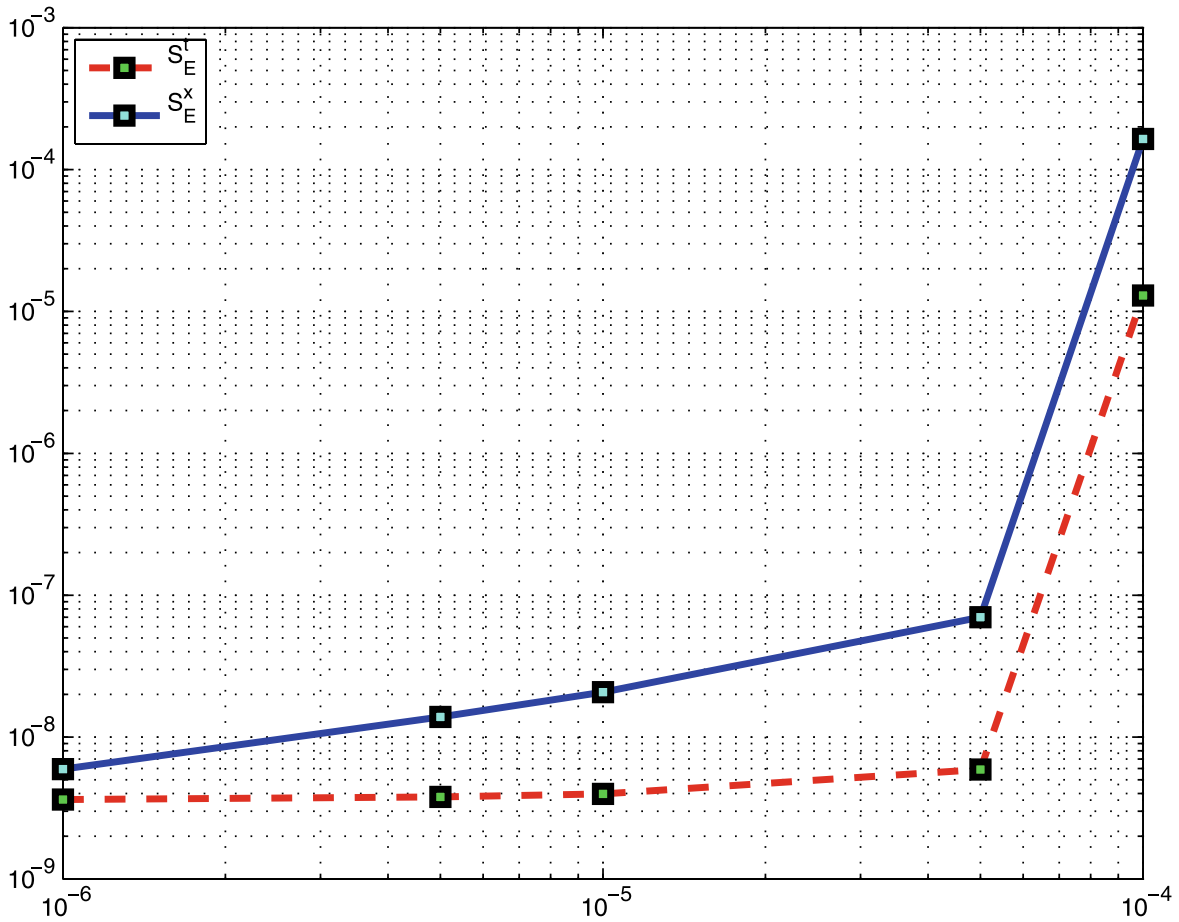

Fig. 6 Test case 1 . Plot of the stability factors $S_{E}^{x}$ and $S_{E}^{t}$ for different values of $\varepsilon$

$t=0.4 T$ and $t=0$ in Figs. 10 and 11 respectively. The behavior of stability factors a function of mesh size for $5 \times 10^{-2} \leq h \leq 5 \times 10^{-4}$ are shown in Fig. 12 .

Note that in our examples, in the first part the related stability factors are almost constant over the whole time interval (Figs. 3 and 9) while in the second part the stability factors tend to increase with increasing (decreasing) viscosity (mesh size) (Figs. 5 and 11). This has confirmed that in a computational approach to stability factors estimation the result can be influenced not only by the geometry and initial 

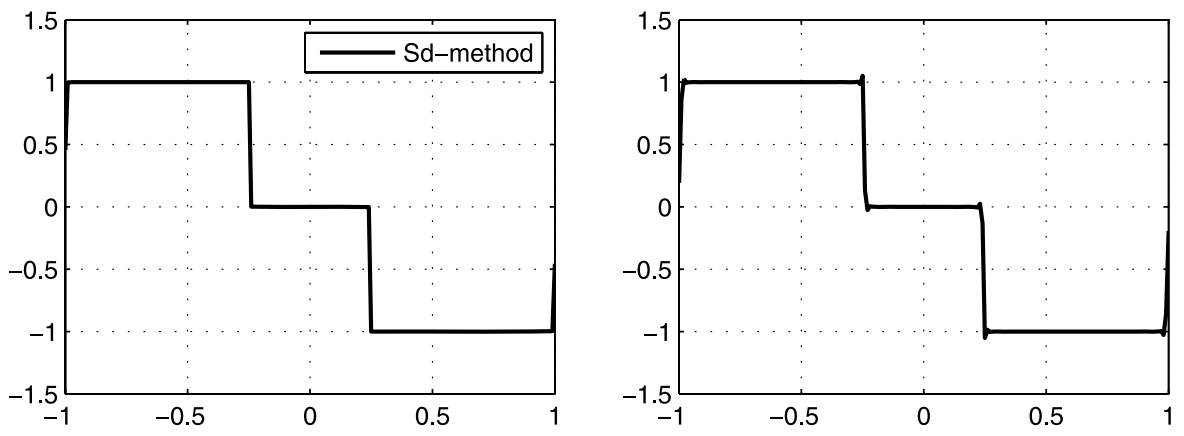

Fig. 7 Test case 2. Plot of $u$ by streamline diffusion method at $t=0$ (left) and $t=T$ (right) for $h=10^{-2}$
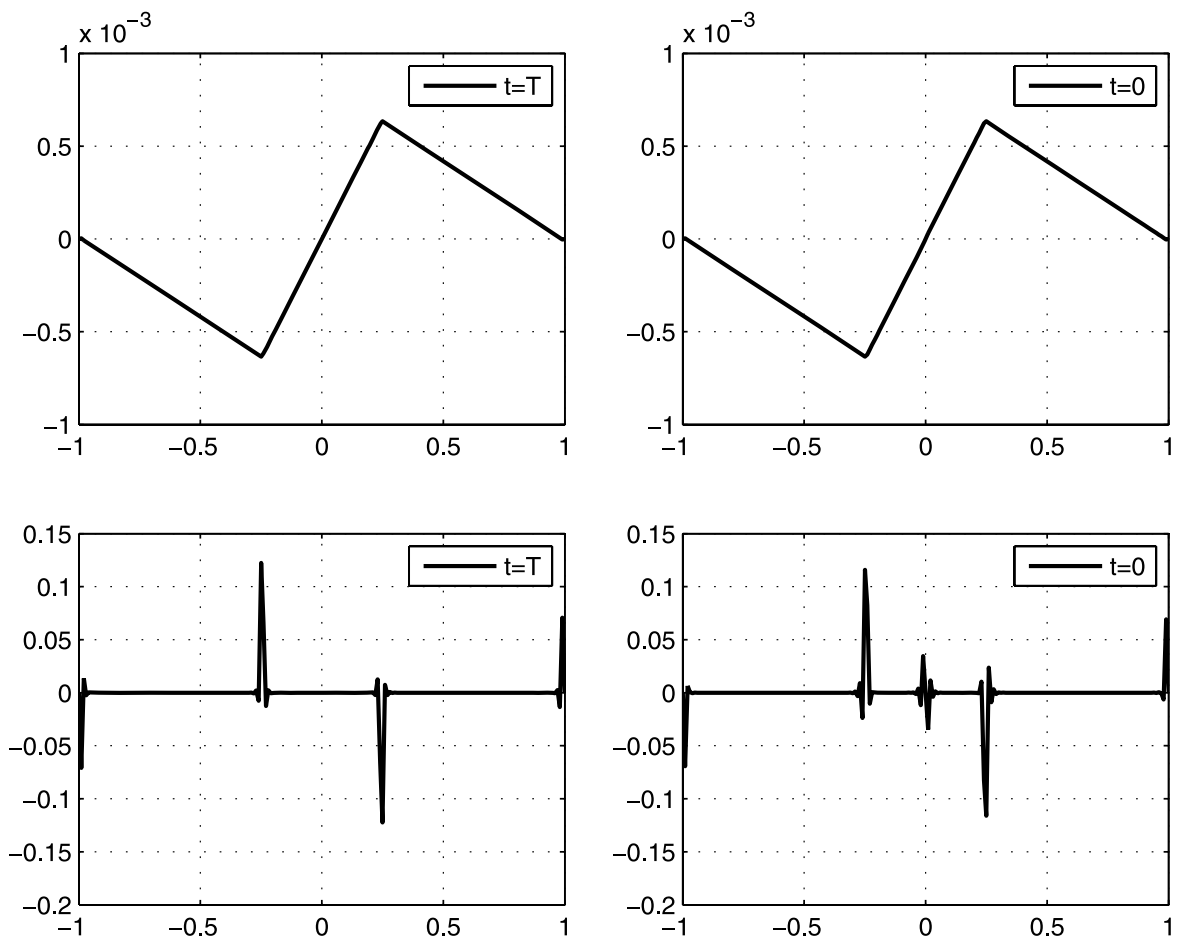

Fig. 8 Test case 2. Plot of $\varphi(t)($ top $)$ and $\varphi_{x x}(t)\left(\right.$ bottom) at $t=T$ and $t=0$ for mesh size $h=10^{-2}$

data given by the problem in question but also by the computational mesh. This is of course an unwanted effect since we would like to compute the stability factors on a relatively coarse mesh to be able to concentrate the computational effort on the actual problem solving [4]. 

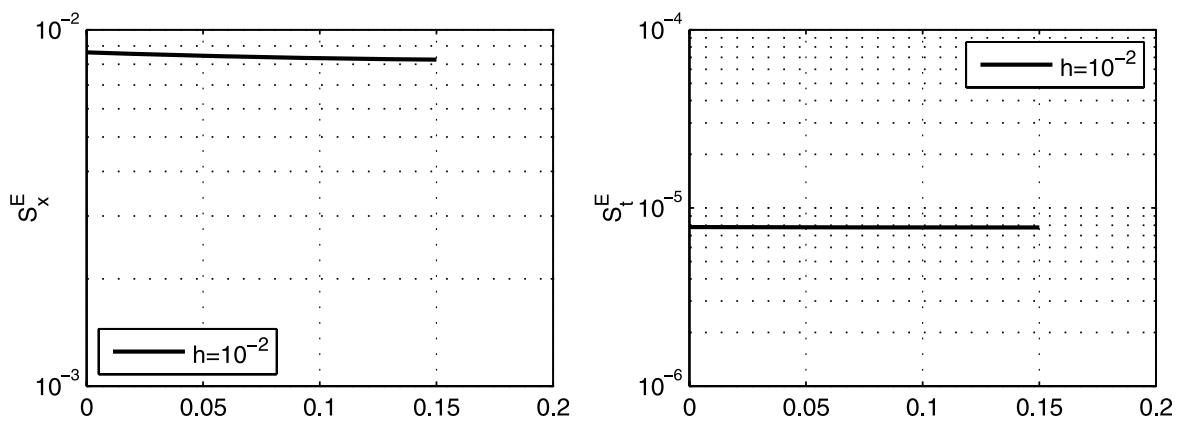

Fig. 9 Test case 2. Plot of the stability factors $S_{E}^{x}$ and $S_{E}^{t}$ versus time for mesh size $h=10^{-2}$
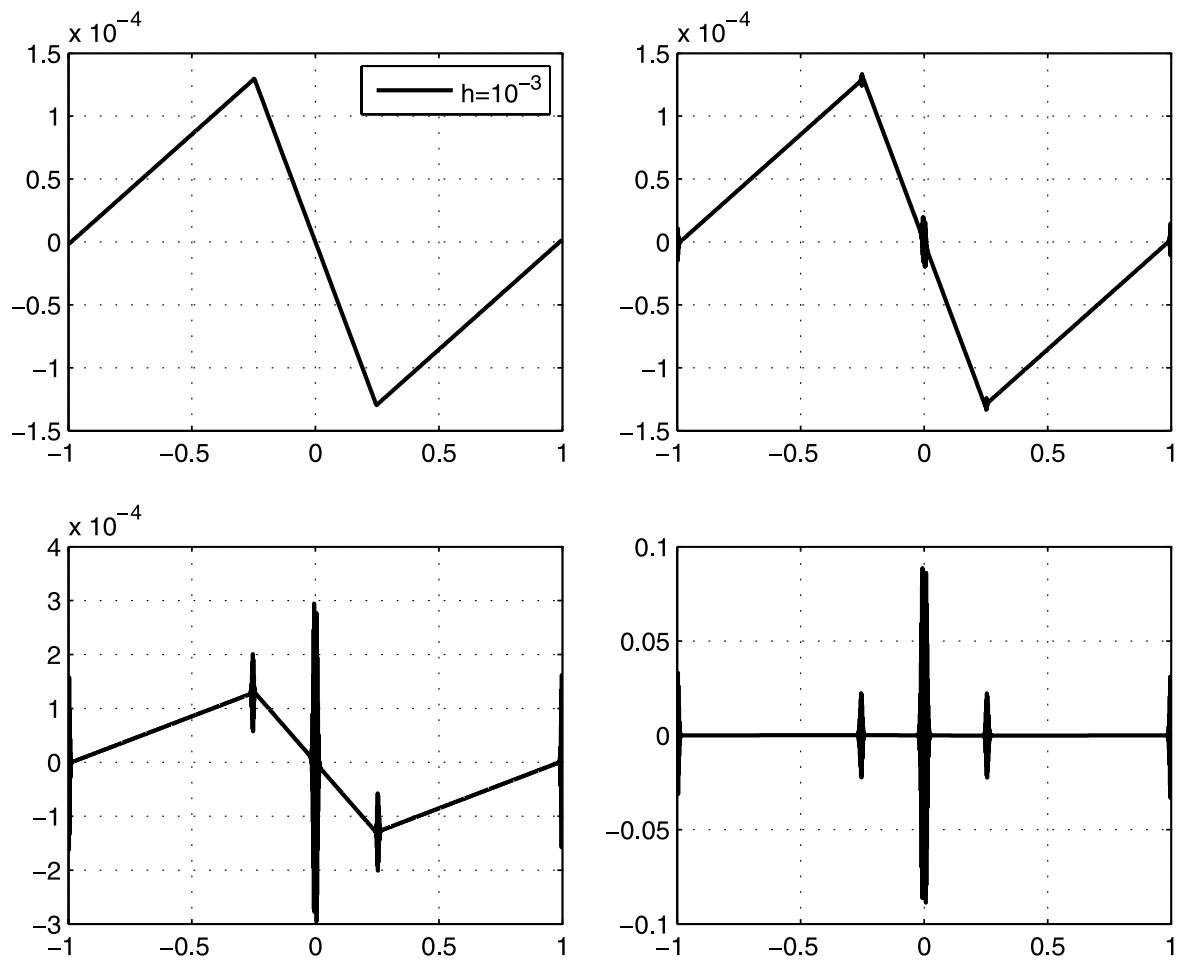

Fig. 10 Test case 2. Plot of $\varphi(t)$ at $t=T, t=0.5 T$ (top), $t=0.4 T$ and $t=0$ (bottom) for mesh size $h=10^{-3}$

\section{Conclusions}

In this note we have focused on the approximation of stability factors for a posteriori error estimation for coupling of two equations analytically and numerically. We have studied the variation of stability factors with different parameters, such as viscosity and mesh size. In the examples 1 we got moderately sized stability factors for small viscosity that these results indicate the possibility of quantitative error control in the 

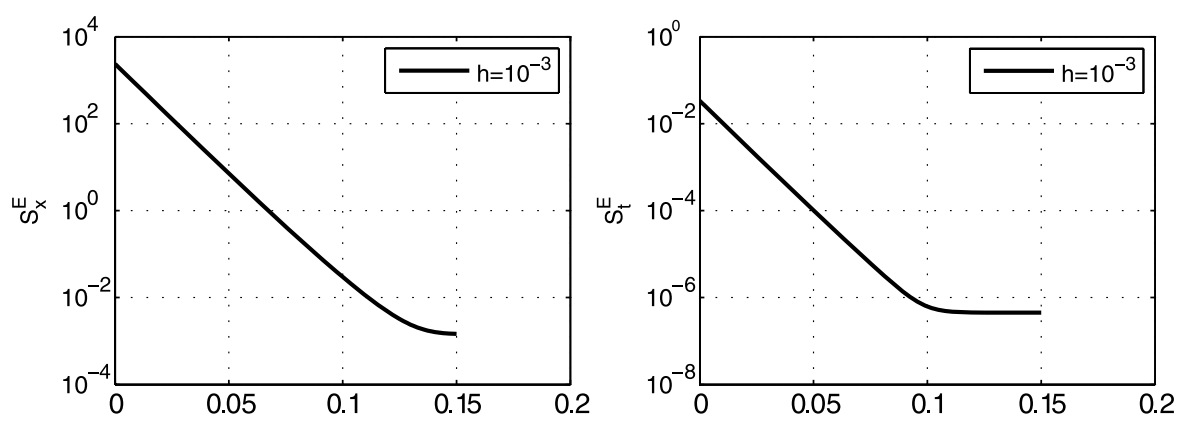

Fig. 11 Test case 2. Plot of the stability factors $S_{E}^{x}$ and $S_{E}^{t}$ versus time for mesh size $h=10^{-3}$

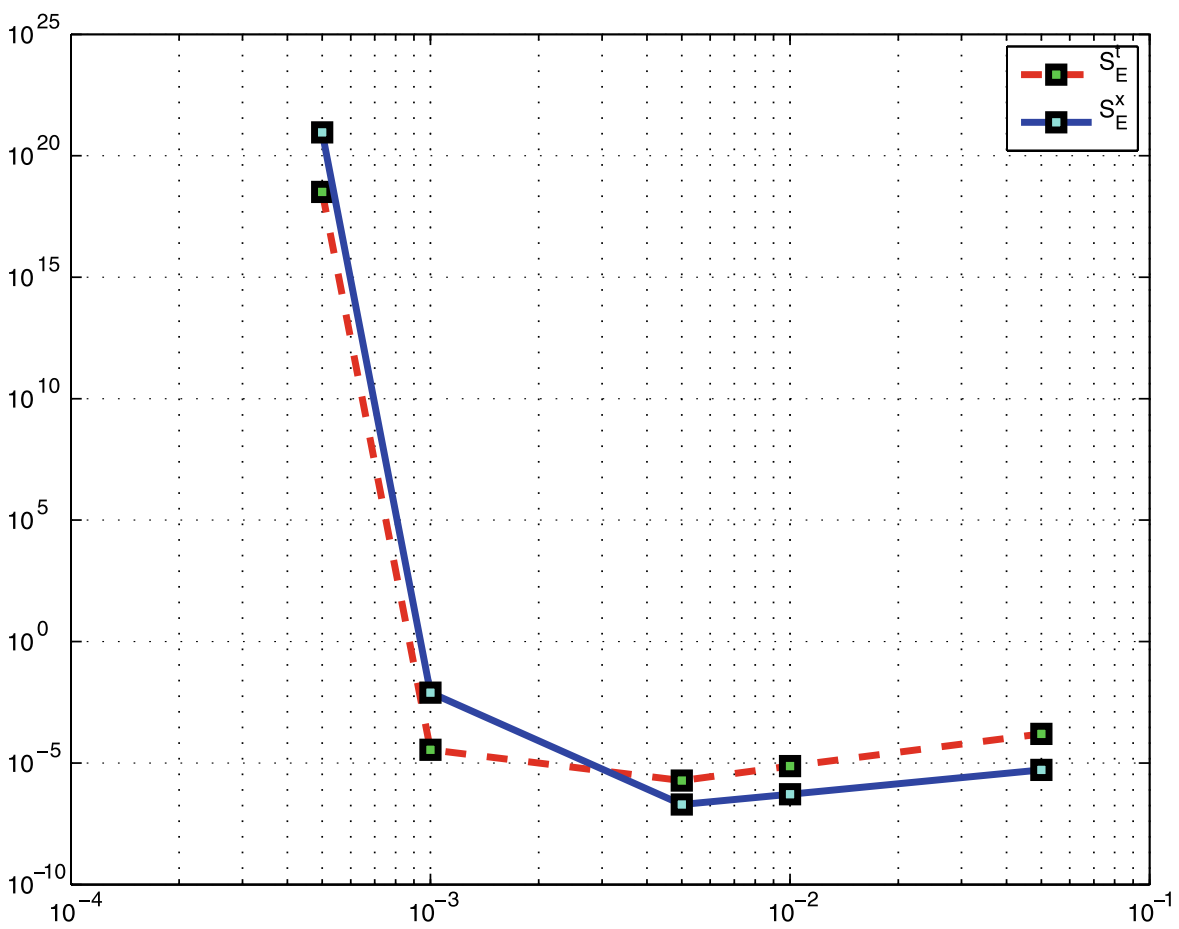

Fig. 12 Test case 2. Plot of the stability factors $S_{E}^{x}$ and $S_{E}^{t}$ for different values of $h$

$H^{-1}$-norm. In example 2 it was noted that the stability factors tend to increase with decreasing mesh size, in other words, the stability factors for $H^{-1}$-norm error control appear to be sensitive to mesh refinement. The result indicate the impossibility of computing stability factors on a coarse mesh and then use them on the finer mesh; of course this is more interesting when there is no analytical solution $u$ and therefore the most fundamental approach for solving dual problem is using two meshes, a fine and coarse mesh for approximating the error by differentiating $u$ on them. 
Acknowledgements This paper is a second part of my thesis work at IASBS. I wish to express my deep thanks to my advisor, Professor Mohammad Asadzadeh, Chalmers University of Technology, for several fruitful comments on this paper.

Open Access This article is distributed under the terms of the Creative Commons Attribution Noncommercial License which permits any noncommercial use, distribution, and reproduction in any medium, provided the original author(s) and source are credited.

\section{References}

1. Asadzadeh, M.: A posteriori error estimates for the Fokker-Planck and Fermi Pencil Beam equations. Math. Models Methods Appl. Sci. 10, 737-769 (2000)

2. Beilina, L., Johnson, C.: A posteriori error estimation in computational inverse scattering. Math. Models Methods Appl. Sci. 15, 23-36 (2005)

3. Brezzi, F., Canuto, C., Russo, A.: A self-adaptive formulation for the Euler/Navier Stokes coupling. Comput. Methods Appl. Mech. Eng. 73, 317-330 (1989)

4. Burman, E.: Adaptive finite element methods for compressible flow. Ph.D. Thesis, Department of Mathematics, Chalmers University of Technology, Göteborg (1998)

5. Ciarlet, P.G.: The Finite Element Method for Elliptic Problems. North-Holland, Amsterdam (1987)

6. Eriksson, K., Johnson, C.: An adaptive finite element method for linear elliptic problems. Math. Comput. 50, 361-383 (1988)

7. Eriksson, K., Johnson, C.: Adaptive finite element methods for parabolic problems I: A linear model problem. SIAM J. Numer. Anal. 28, 43-77 (1991)

8. Eriksson, K., Johnson, C.: Adaptive finite element methods for parabolic problems II: optimal error estimates in $L_{\infty} L_{2}$ and $L_{\infty} L_{\infty}$. SIAM J. Numer. Anal. 32, 706-740 (1995)

9. Eriksson, K., Johnson, C.: Adaptive finite element methods for parabolic problems IV: non-linear problems. SIAM J. Numer. Anal. 32, 777-788 (1995)

10. Godlewski, E., Raviart, P.-A.: The numerical interface coupling of nonlinear hyperbolic systems of conservation laws: I. The scalar case. Numer. Math. 97, 81-130 (2004)

11. Houston, P., Süli, E.: Adaptive Lagrange-Galerkin methods for unsteady convection-dominated diffusion problems. Math. Comput. 70, 77-106 (2001)

12. Izadi, M.: Streamline diffusion method for treating the coupling equations of two hyperbolic conservation laws. Math. Comput. Model. 45, 201-214 (2007)

13. Johnson, C., Szepessy, A.: Adaptive finite element methods for conservation laws based on a posteriori error estimates. Commun. Pure Appl. Math. 48, 199-234 (1995)

14. Johnson, C., Szepessy, A., Hansbo, P.: On the convergence of shock-capturing streamline diffusion finite element methods for hyperbolic conservation laws. Math. Comput. 54, 107-129 (1990)

15. Sandboge, R.: Adaptive finite element methods for reactive flow problems. Ph.D. Thesis, Department of Mathematics, Chalmers University of Technology, Göteborg (1996)

16. Süli, E., Houston, P.: Finite element methods for hyperbolic problems: a posteriori error analysis and adaptivity. In: Duff, I., Watson, G.A. (eds.) State of the Art in Numerical Analysis, pp. 441-471. Oxford University Press, Oxford (1997)

17. Szepessy, A.: Convergence of streamline diffusion finite element method for conservation law. Ph.D. Thesis, Department of Mathematics, Chalmers University of Technology, Göteborg (1989) 